\title{
Absolute stability and synchronization in neural field models with transmission delays
}

\author{
Chiu-Yen Kao ${ }^{\mathrm{a}}$, Chih-Wen Shih ${ }^{\mathrm{b}, *}$, Chang-Hong Wu ${ }^{\mathrm{c}}$ \\ ${ }^{a}$ Department of Mathematical Sciences, Claremont McKenna College, Claremont, CA 91711, USA \\ ${ }^{b}$ Department of Applied Mathematics, National Chiao Tung University, Hsinchu 300, Taiwan \\ ${ }^{c}$ Department of Applied Mathematics, National University of Tainan, Tainan 700, Taiwan
}

\begin{abstract}
Neural fields model macroscopic parts of the cortex which involve several populations of neurons. We consider a class of neural field models which are represented by integro-differential equations with transmission time delays which are space-dependent. The considered domains underlying the systems can be bounded or unbounded. A new approach, called sequential contracting, instead of the conventional Lyapunov functional technique, is employed to investigate the global dynamics of such systems. Sufficient conditions for the absolute stability and synchronization of the systems are established. Several numerical examples are presented to demonstrate the theoretical results.
\end{abstract}

Keywords: absolute stability, synchronization, neural field models, delay equations

\section{Introduction}

Neural fields are neural continuum networks which are proposed to model macroscopic parts of the cortex at population level. Since the pioneering works of Wilson and Cowan [37, 38] and Amari [1, 2], there have been tremendous efforts towards developing mathematical tools to investigate neural field models. These models are typically in the form of integro-differential equations and have revealed very rich dynamics such as traveling wavefronts, traveling pulses and stable localized stationary solutions, see, for example, [5, 10, 14, 15, 22, 23, 24, 25, 27], and the review articles $[6,7,9]$. Neural field models have been adopted to depict brain rhythmic activity [13, 20,31]. More realistic applications can be found in [16, 34].

Recently, neural field models which take into account transmission time delays have received considerable attention. In such models, for $N$ populations of neurons which occupy spatial domain $\Omega \subseteq \mathbf{R}^{n}$, the average membrane potential $V_{i}(x, t)$ of the $i$ th population positioned at $x \in \Omega$ satisfies

$$
\frac{\partial V_{i}(x, t)}{\partial t}=-\frac{1}{l_{i}} V_{i}(x, t)+\sum_{j=1}^{N} \int_{\Omega} W_{i j}(x, y, t) S_{j}\left(V_{j}\left(y, t-\tau_{j}(x, y)\right)\right) d y+I_{i}(x, t)
$$

at time $t$, where $i=1, \ldots, N$. Herein, $l_{i}>0$ characterize the activity decay of the $i$ th population; the connectivity function $W_{i j}(x, y, t)$ describe how the populations at $y$ influence those at $x$ at time $t ; S_{i}\left(V_{i}(x, t)\right)$ stand for the activation function for interacting neurons; $I_{i}(x, t)$ are external currents; $\tau_{i}(x, y) \geq 0$ measure the propagation delays which are space-dependent. A reasonable choice of $\tau_{i}(x, y)$ is, for example, $\tau_{i}(x, y):=\|x-y\| / c_{i}$ for some $c_{i}>0, i=1, \ldots, N$. If $\Omega$ is a bounded domain in $\mathbf{R}^{n}$, then each $\tau_{i}$ is a bounded function. In this case, there exists a positive constant $\tau^{M}$ defined by

$$
\tau^{M}:=\max _{i=1, \ldots, N} \sup _{x, y \in \Omega} \tau_{i}(x, y)
$$

\footnotetext{
${ }^{*}$ Corresponding author

Email addresses: Chiu-Yen.Kao@ClaremontMcKenna.edu (Chiu-Yen Kao), cwshih@math.nctu.edu.tw (Chih-Wen Shih), changhong@mail.nutn.edu.tw (Chang-Hong Wu)
}

Preprint submitted to Elsevier

February 24, 2016 
One can also write (1) into vector form:

$$
\frac{\partial \mathbf{V}(x, t)}{\partial t}=-\mathbf{L V}(x, t)+\int_{\Omega} \mathbf{W}(x, y, t) \mathbf{S}(\mathbf{V}(y, t-\tau(x, y))) d y+\mathbf{I}(x, t),
$$

where $\mathbf{V}=\left(V_{1}, \ldots, V_{N}\right)^{T}, \mathbf{L}=\operatorname{diag}\left(1 / l_{i}\right), \mathbf{W}=\left[W_{i j}\right]_{1 \leq i, j \leq N}, \mathbf{S}(\mathbf{V}):=\left(S_{1}\left(V_{1}\right), \ldots, S_{N}\left(V_{N}\right)\right)^{T}, \mathbf{I}=\left(I_{1}, \ldots, I_{N}\right)^{T}, \tau=$ $\left(\tau_{1}, \ldots, \tau_{N}\right)$, and we interpret

$$
\mathbf{S}(\mathbf{V}(y, t-\tau(x, y)))=\left(S_{1}\left(V_{1}\left(y, t-\tau_{1}(x, y)\right)\right), \ldots, S_{N}\left(V_{N}\left(y, t-\tau_{N}(x, y)\right)\right)\right)^{T} .
$$

Following the setting in the fundamental theory of delay equations [18], we shall consider the evolution of membrane potential $\mathbf{V}(x, t)$ according to (3) from given initial data $\phi$, i.e.,

$$
\mathbf{V}(x, t)=\phi(x, t), x \in \Omega, t \in\left[-\tau^{M}, 0\right] .
$$

System (1) is regarded as the voltage-based model and its counterpart, the activity-based model (without delays) was depicted in [13]. Derivation of (1) with $N=1$ using the impulse response function as a Green's function has been summarized in [3]. Developing fundamental theory for system (1), an integro-differential equation with spacedependent time delay, is a nontrivial task. In delay equation setting, the phase space is typically $C:=C\left(\left[-\tau^{M}, 0\right] ; \mathcal{X}\right)$. For a bounded and connected $\Omega \subset \mathbf{R}^{n}$, Van Gils et al. [32] chose $\mathcal{X}=C(\bar{\Omega})$ which fits in the theory of dual semigroups well and meets the modelling need. It also led to set up an analytic framework for the study of stability and bifurcation of steady states for system (1). Under this framework, the characteristic equation for an example of system (1) was derived and Hopf bifurcation was discussed. In [12], Faye and Faugeras constructed a Lyapunov functional and derived a sufficient condition for uniformly asymptotical stability of the origin for the linearized system of (1) at a stationary solution, when the connectivity and the external inputs are time-independent, i.e., $\mathbf{W}(x, y, t)=\mathbf{W}(x, y)$ and $\mathbf{I}(x, t)=\mathbf{I}(x)$, for all $t \geq 0$.

When $\tau^{M}=0$ (delay being neglected) and $\Omega$ is a compact subset of $\mathbf{R}^{n}$, system (3) reduces to the one studied in [13]. The existence and uniqueness of classical solutions were established therein. In addition, a sufficient condition for the absolute stability of the general solution was provided using the Lyapunov functional technique. By absolute stability of the general solution or of the system, it means that any two solutions approach each other as $t \rightarrow \infty$, regardless of their initial data. This notion is associated with the neuronal dynamics in the sense that absolutely stable system evolves to a state which only depends on the input, not the initial state. Such systems are able to differentiate distinct stimuli by converging to corresponding states without hinging upon initial data. Synchronization for (3) was also addressed in [13], where synchronization means that all homogeneous (space-independent) solutions of (3) converge to the unique homogeneous solution which varies with respect to the space-independent input $\mathbf{I}=\mathbf{I}(t)$ and not on the initial state.

When $\Omega=\mathbf{R}^{n}$, this model (3) can be regarded as a generalization of Amari's model [2], where the space-dependent propagation delays were not taken into account. Although considering the infinite domain may not be biologically realistic, it is more convenient mathematically, to investigate various wave solutions or spatiotemporal patterns when $\Omega=\mathbf{R}^{n}$, see, for example, $[3,4,33]$ and the references therein.

In this paper, we shall study (3) and (4) on a bounded or unbounded domain $\Omega$ in $\mathbf{R}^{n}$. If $\Omega$ is unbounded, then we assume that $\tau$ is an increasing function of $\|x-y\|$ with a finite supremum $\tau^{M}:=\max _{i=1, \ldots, N} \sup _{x, y \in \Omega} \tau_{i}(x, y)$. We develop an approach disparate from Lyapunov functional method to conclude the global dynamics in the delay integrodifferential equation (3) on both bounded and unbounded domains. In particular, we shall derive the criteria for the absolute stability and global synchronization for the systems. It turns out that we are able to extend the theory of absolute stability for the system without delay, reported in [13], to time-delay cases and can also handle the models on whole space domain. To this end, we shall consider solutions more regular than the ones in [12]. Indeed, we shall focus on the solutions which are bounded and continuous in $\Omega$ and continuously differentiable in $t \geq 0$.

The rest of this paper is organized as follows. In Section 2, we introduce some function spaces to be used later and prove the existence and uniqueness of solution for (3) and (4). In Section 3, we introduce a methodology called sequential contracting to investigate the stability and synchronization. Section 4 is devoted to the absolute stability and synchronization of solutions for system (3). In Section 5, we provide some numerical simulations and examples. Finally, we give a brief conclusion in Section 6. 


\section{Initial value problem}

In this section, we shall study the existence and uniqueness of solution to the initial value problem (3) and (4), where the domain $\Omega \subseteq \mathbf{R}^{n}$ can be bounded or unbounded. Hereafter, the $L^{p}$ norm of a vector-valued function $\mathbf{g}=$ $\left(g_{1}, \ldots, g_{N}\right), 1 \leq p<\infty$, is defined by

$$
\|\mathbf{g}\|_{L^{p}(\Omega)}:=\max _{i=1, \ldots, N}\left\|g_{i}\right\|_{L^{p}(\Omega)},
$$

and we denote by $L^{p}\left(\Omega ; \mathbf{R}^{N}\right.$ ) (in short, $L^{p}(\Omega)$ ) the set of functions with finite norm. Similarly, the supremum norm of a vector-valued function $\mathbf{g}=\left(g_{1}, \ldots, g_{N}\right)$ is given by

$$
\|\mathbf{g}\|_{\infty}=\max _{i=1, \ldots, N}\left\|g_{i}\right\|_{\infty}:=\max _{i=1, \ldots, N} \sup _{x \in \Omega}\left|g_{i}(x)\right| .
$$

The $L^{p}$ norm of a $n \times n$ matrix function $\mathbf{w}=\left[w_{i j}\right]$ is defined by

$$
\|\mathbf{w}\|_{L^{p}(\Omega)}:=\max _{i=1, \ldots, N} \sum_{j=1}^{N}\left\|w_{i j}\right\|_{L^{p}(\Omega) .} .
$$

Our approach to studying the existence and uniqueness of solution in the delayed neural field system (3)-(4) is similar to the one in [12]. However, the function spaces we choose here are different and the connectivity function $\mathbf{W}$ is assumed to be more regular than the one in [12] so that the present approach can treat unbounded domain $\Omega$. In addition, our methodology for establishing the absolute stability of solutions, presented in the next section, requires continuous solutions of (3). Therefore, the solutions we consider are continuous in $x$ and continuously differentiable in $t$.

We now define the Banach space $\mathcal{X}:=B C\left(\Omega ; \mathbf{R}^{N}\right)$ of bounded and continuous functions mapping $\Omega$ into $\mathbf{R}^{N}$ with the norm

$$
\|\mathbf{u}\|_{X}:=\max _{i=1, \ldots, N}\left\|u_{i}\right\|_{\infty}=\max _{i=1, \ldots, N} \sup _{x \in \Omega}\left|u_{i}(x)\right| \quad \text { for } \mathbf{u}=\left(u_{1}, \ldots, u_{N}\right) .
$$

For a given $\alpha \in(0,1)$, we introduce the function space $\mathcal{Y}_{\alpha}:=B C^{\alpha}\left(\Omega ; L^{1}(\Omega)\right)$ consisting of $N \times N$ matrix functions $\mathbf{w}(x, y)=\left[w_{i j}(x, y)\right]$ defined on $\Omega \times \Omega$, with the norm (cf. [28])

$$
\|\mathbf{w}\|_{y_{\alpha}}:=\sup _{x \in \Omega}\|\mathbf{w}(x, \cdot)\|_{L^{1}(\Omega)}+\sup _{x, \hat{x} \in \Omega, x \neq \hat{x}} \frac{\|\mathbf{w}(x, \cdot)-\mathbf{w}(\hat{x}, \cdot)\|_{L^{1}(\Omega)}}{\|x-\hat{x}\|^{\alpha}},
$$

where $\|x-\hat{x}\|:=\max \left\{\left|x_{1}-\hat{x}_{1}\right|, \ldots,\left|x_{n}-\hat{x}_{n}\right|\right\}$ for $x=\left(x_{1}, \ldots, x_{n}\right)$ and $\hat{x}=\left(\hat{x}_{1}, \ldots, \hat{x}_{n}\right)$.

We shall consider the phase space

$$
C:=C\left(\left[-\tau^{M}, 0\right] ; \mathcal{X}\right),
$$

the continuous functions from time interval $\left[-\tau^{M}, 0\right]$ to $\mathcal{X}$ with the norm

$$
\|\phi\|_{C}:=\sup _{\theta \in\left[-\tau^{M}, 0\right]}\|\phi(\theta)\|_{X} .
$$

Then, given an initial value $\phi \in C$, we consider the initial value problem for a retarded functional differential equation on $X$ :

$$
\dot{\mathbf{V}}=\mathbf{F}\left(t, \mathbf{V}_{t}\right), \quad \mathbf{V}_{0}=\phi \in C,
$$

where

$$
\mathbf{F}\left(t, \mathbf{V}_{t}\right)(x):=-\mathbf{L V}_{t}(0)(x)+\int_{\Omega} \mathbf{W}(x, y, t) \mathbf{S}\left(\mathbf{V}_{t}(-\tau(x, y))(y)\right) d y+\mathbf{I}(x, t)
$$

for $x \in \Omega$ and $t \geq 0$. Here $\mathbf{V}_{t}(\theta)(x)=\mathbf{V}(x, t+\theta)$, for $\theta \in\left[-\tau^{M}, 0\right]$.

We now present the existence and uniqueness of solution for (5). Let $\left[0, \tau^{M}\right]^{N}$ denote the product of $N\left[0, \tau^{M}\right]$ 
Theorem 2.1. Assume that the following assumptions hold:

(Al) $\mathbf{S} \in B C^{1}\left(\mathbf{R}^{n} ; \mathbf{R}^{N}\right)$,

(A2) $\tau \in C\left(\Omega \times \Omega ;\left[0, \tau^{M}\right]^{N}\right)$, and the continuity is uniform on $\Omega \times \Omega$,

(A3) $\mathbf{W} \in C\left(\left[-\tau^{M}, \infty\right) ; \mathcal{Y}_{\alpha}\right)$,

(A4) $\mathbf{I} \in C\left(\left[-\tau^{M}, \infty\right) ; \mathcal{X}\right)$.

Then (5) has a unique solution $\mathbf{V}$ which is continuous in $t$ for $t \in\left[-\tau^{M}, \infty\right)$ and continuously differentiable in $t$ for $t \in[0, \infty)$, i.e.,

$$
\mathbf{V} \in C^{1}([0, \infty) ; \mathcal{X}) \cap C\left(\left[-\tau^{M}, \infty\right) ; \mathcal{X}\right)
$$

Proof. As in [12], we shall apply the Cauchy-Lipschitz theorem for retarded functional differential equations on Banach space to prove the local existence and uniqueness. First, we observe that $\mathbf{F}$ maps $J \times C$ into $\mathcal{X}$, where $J:=\left[-\tau^{M}, t_{1}\right]$ for a given $t_{1}>0$. Indeed, for a given $(t, \psi) \in J \times C$, by assumption we have

$$
\left\|\int_{\Omega} \mathbf{W}(x, y, t) \mathbf{S}(\psi(y,-\tau(x, y))) d y\right\| \leq\|\mathbf{S}\|_{\infty}\|\mathbf{W}(t)\|_{y_{\alpha}} \quad \text { for all } x \in \Omega
$$

Thus, we see that $\mathbf{F}$ is bounded on $\Omega$ for each $(t, \psi) \in J \times C$. To prove the continuity, we first focus on the integral term of (6). For any given $x, \hat{x} \in \Omega$,

$$
\begin{aligned}
& \left\|\int_{\Omega} \mathbf{W}(x, y, t) \mathbf{S}(\psi(y,-\tau(x, y))) d y-\int_{\Omega} \mathbf{W}(\hat{x}, y, t) \mathbf{S}(\psi(y,-\tau(\hat{x}, y))) d y\right\| \\
& \leq\left\|\int_{\Omega} \mathbf{W}(x, y, t) \mathbf{S}(\psi(y,-\tau(x, y))) d y-\int_{\Omega} \mathbf{W}(\hat{x}, y, t) \mathbf{S}(\psi(y,-\tau(x, y))) d y\right\| \\
& +\left\|\int_{\Omega} \mathbf{W}(\hat{x}, y, t) \mathbf{S}(\psi(y,-\tau(x, y))) d y-\int_{\Omega} \mathbf{W}(\hat{x}, y, t) \mathbf{S}(\psi(y,-\tau(\hat{x}, y))) d y\right\| \\
& \leq\|\mathbf{S}\|_{\infty}\|\mathbf{W}(x, \cdot, t)-\mathbf{W}(\hat{x}, \cdot, t)\|_{L^{1}(\Omega)} \\
& +\left\|\mathbf{S}^{\prime}\right\|_{\infty} \int_{\Omega}\|\mathbf{W}(\hat{x}, y, t)\|\|\psi(y,-\tau(x, y))-\psi(y,-\tau(\hat{x}, y))\| d y \\
& \leq\|\mathbf{S}\|_{\infty}\|\mathbf{W}(t)\| y_{\alpha}\|x-\hat{x}\|^{\alpha} \\
& +\left\|\mathbf{S}^{\prime}\right\|_{\infty} \int_{\Omega}\|\mathbf{W}(\hat{x}, y, t)\|\|\psi(y,-\tau(x, y))-\psi(y,-\tau(\hat{x}, y))\| d y
\end{aligned}
$$

for $x, \hat{x} \in \Omega$ and $t \in J$, where $\mathbf{S}^{\prime}=\left(S_{1}^{\prime}, \ldots, S_{N}^{\prime}\right)$. By assumptions (A1)-(A3), we see that the integral term of (6) is continuous on $\Omega$. Together with (A4), it follows that $\mathbf{F}(t, \psi)$ is continuous on $\Omega$. Thus, we have proved that $\mathbf{F}$ maps $J \times C$ into $\mathcal{X}$.

To apply the Cauchy-Lipschitz theorem, it suffices to show that

(i) $\mathbf{F}$ is continuous with respect to $(t, \psi)$ in each compact set in $J \times C$;

(ii) $\mathbf{F}$ is Lipschitz continuous with respect to its second argument in each compact set in $J \times C$.

For (i), observe that

$$
\begin{aligned}
\mathbf{F}\left(t, \psi_{1}\right)(x)-\mathbf{F}\left(s, \psi_{2}\right)(x)= & -\mathbf{L}\left[\psi_{1}(x, 0)-\psi_{2}(x, 0)\right] \\
& +\int_{\Omega}[\mathbf{W}(x, y, t)-\mathbf{W}(x, y, s)] \mathbf{S}\left(\psi_{1}(y,-\tau(x, y)) d y\right. \\
& +\int_{\Omega} \mathbf{W}(x, y, s)\left[\mathbf{S}\left(\psi_{1}(y,-\tau(x, y))\right)-\mathbf{S}\left(\psi_{2}(y,-\tau(x, y))\right)\right] d y \\
& +\mathbf{I}(x, t)-\mathbf{I}(x, s) .
\end{aligned}
$$


Then we have

$$
\begin{aligned}
\left\|\mathbf{F}\left(t, \psi_{1}\right)-\mathbf{F}\left(s, \psi_{2}\right)\right\|_{\mathcal{X}} \leq & \|\mathbf{L}\|_{\infty}\left\|\psi_{1}-\psi_{2}\right\|_{C}+\|\mathbf{S}\|_{\infty} \sup _{x \in \Omega}\|\mathbf{W}(x, \cdot, t)-\mathbf{W}(x, \cdot, s)\|_{L^{1}(\Omega)} \\
& +\left\|\mathbf{S}^{\prime}\right\|_{\infty}\|\mathbf{W}(s)\|_{y_{\alpha}}\left\|\psi_{1}-\psi_{2}\right\|_{C}+\|\mathbf{I}(t)-\mathbf{I}(s)\|_{\mathcal{X}},
\end{aligned}
$$

where $\|\mathbf{L}\|_{\infty}:=\max _{i=1, \ldots, N} l_{i}^{-1}$. By assumptions (A1)-(A4), we have justified the continuity of $\mathbf{F}$, which in turn implies (i). Furthermore, putting $s=t$ into the above inequality yields

$$
\left\|\mathbf{F}\left(t, \psi_{1}\right)-\mathbf{F}\left(t, \psi_{2}\right)\right\|_{\mathcal{X}} \leq\|\mathbf{L}\|_{\infty}\left\|\psi_{1}-\psi_{2}\right\|_{C}+\left\|\mathbf{S}^{\prime}\right\|_{\infty}\|\mathbf{W}(t)\|_{y_{\alpha}}\left\|\psi_{1}-\psi_{2}\right\|_{C} .
$$

Again, (ii) follows from assumptions (A1)-(A4).

From (i) and (ii), we obtain the local existence and uniqueness of solution. In fact, the solution can be extended to all forward time. That is, there exists a unique solution $\mathbf{V}$ of (5) with

$$
\mathbf{V} \in C^{1}([0, \infty) ; \mathcal{X}) \cap C\left(\left[-\tau^{M}, \infty\right) ; \mathcal{X}\right)
$$

This can be justified by a process similar to the proof of [12, Theorem 3.2.1]. We thus complete the proof.

Remark 2.1. The existence and uniqueness of solution for (3) and (4) were reported in [12], where $X$ is chosen as $L^{2}\left(\Omega ; \mathbf{R}^{N}\right)$ with bounded $\Omega \subset \mathbf{R}^{n}$. On the other hand, it was pointed out in [32] that some difficulties arise with such a choice of $\mathcal{X}$, including the definition of the integral operator $G$ associated with the integral term in (3) and the Fréchet differentiability of $G$. Instead, $\mathcal{X}=C(\bar{\Omega})$ was chosen in [32], and the wellposedness of (3) and (4) in $C\left(\left[-\tau^{M}, 0\right] ; C(\bar{\Omega})\right)$ and the global solution were put in the framework of delay differential equations and the theory of dual semigroups. This framework then led to the study of stability and bifurcation of steady states for system (3). When the domain $\Omega \subseteq \mathbf{R}^{n}$ is bounded and satisfies the cone property, under assumption less regular than the present one, Veltz and Faugeras [35] proved the existence and uniqueness of solution for (3) and (4) in $C\left([0, T] ; W^{k, 2}(\Omega)\right)$ for each $T>0$. By the embedding theorem, their solutions actually belong to $C([0, T] ; C(\bar{\Omega}))$ if $k$ is large enough. When $\Omega=\mathbf{R}^{n}, N=1, \tau^{M}=0$ (i.e., without time delays) and the connectivity matrix $\mathbf{W}$ is independent of $t$, the existence and uniqueness of solutions were proved in [28].

We end this section with a fundamental property of continuous dependence on initial data.

Proposition 2.1. Let $\mathbf{V}$ be the solution of (3) with initial data

$$
\mathbf{V}(x, t)=\phi^{V}(t)(x), x \in \Omega, t \in\left[-\tau^{M}, 0\right] .
$$

Then for any given $t_{1}>0$ and $\varepsilon>0$, there exists a $\delta>0$ depending only on $t_{1}$ and $\varepsilon$ such that $\|\mathbf{V}(\cdot, t)-\mathbf{U}(\cdot, t)\|_{\infty}<\varepsilon$ for all $t \in\left[0, t_{1}\right]$, for any solution $\mathbf{U}$ of (3) with initial data $\phi^{U}$ satisfying $\sup _{\theta \in\left[-\tau^{M}, 0\right]}\left\|\phi^{V}(\theta)-\phi^{U}(\theta)\right\|_{\infty}<\delta$.

Proof. The proof follows by applying an argument similar to the proof of Theorem 2.2 in [18].

\section{Sequential contracting}

In this section, we shall present the approach called sequential contracting to investigate the absolute stability and synchronization for the neural field models (3). The idea is to establish an iteration scheme so that the behavior of the difference of two arbitrary solutions can be estimated. Such an idea was first proposed to study asymptotic behaviors in a class of difference-differential systems in [29, 30].

We denote by $C^{0,1}(\Omega \times[0, \infty) ; \mathbf{R})$ the space consisting of functions continuous on $\Omega$ and continuously differentiable in $t \geq 0$. For an $r \geq 0$, we denote $D_{r}:=\{(x, t): x \in \Omega, t \geq r\}$.

Lemma 3.1. Assume that for a real-valued function $u \in C^{0,1}\left(D_{t_{0}}\right)$, there exists a $M>0$ such that

$$
|u(x, t)| \leq M \quad \text { for all }(x, t) \in D_{t_{0}} .
$$


If $u$ satisfies

$$
\left|\frac{\partial u(x, t)}{\partial t}+\frac{1}{l} u(x, t)\right| \leq b \quad \text { in } D_{t_{0}} \quad \text { for some } l, b>0,
$$

then for each $\varepsilon>0$, there exists a $T=T\left(\varepsilon, t_{0}\right)>t_{0}$ such that

$$
\|u(\cdot, t)\|_{\infty} \leq b l+\varepsilon \text { for all } t \geq T .
$$

Proof. For an $\varepsilon>0$, from (7), we observe that

$$
\frac{\partial u(x, t)}{\partial t}>\frac{\varepsilon}{l} \quad \text { if } u(x, t)<-b l-\varepsilon, \text { while } \frac{\partial u(x, t)}{\partial t}<-\frac{\varepsilon}{l} \quad \text { if } u(x, t)>b l+\varepsilon .
$$

Thus, $u$ is strictly increasing (resp., decreasing) in time if $u \in(-\infty,-b l-\varepsilon]$ (resp., $u \in[b l+\varepsilon, \infty)$ ). Combining this with the uniform boundedness of $u$, for any given $\varepsilon>0$, we can find a $T=T\left(\varepsilon, t_{0}\right)>t_{0}$ such that $u$ must enter the interval $[-b l-\varepsilon, b l+\varepsilon]$ for all $t \geq T$. This completes the proof.

Lemma 3.2. Consider a vector-valued function $\mathbf{u} \in C^{0,1}\left(D_{t_{0}} ; \mathbf{R}^{N}\right)$ with $\mathbf{u}(x, t)=\left(u_{1}(x, t), \ldots, u_{N}(x, t)\right)$. If there exists $M>0$ such that

$$
\left|u_{i}(x, t)\right| \leq M \quad \text { for all }(x, t) \in D_{t_{0}} \text { and } i=1, \ldots, N
$$

and $\mathbf{u}$ satisfies

$$
\left|\frac{\partial u_{i}(x, t)}{\partial t}+\frac{1}{l} u_{i}(x, t)\right| \leq \beta \sup _{t \geq s}\|\mathbf{u}(\cdot, t)\|_{\infty}+\omega(t)
$$

over $D_{s+r}$ for all $s \geq t_{0}, i=1, \ldots, N$, for some positive constants $\beta, l, r$, and a function $\omega:\left[t_{0}, \infty\right) \rightarrow \mathbf{R}^{+}$, then

$$
\|\mathbf{u}(\cdot, t)\|_{\infty} \rightarrow\left[0, \frac{l A}{1-l \beta}\right] \quad \text { as } t \rightarrow \infty
$$

as long as $\beta l<1$, where

$$
A:=\limsup _{t \rightarrow \infty} \omega(t) \geq 0
$$

Proof. Since $\beta l<1$, we can choose a small $\epsilon>0$ such that

$$
\beta l(1+\epsilon)<1
$$

and we can choose a $\tilde{t}>t_{0}$ such that $\omega(t)<A+\epsilon$ for all $t \geq \tilde{t}$, due to (11). By Lemma 3.1, there exists a $t_{1}>\tilde{t}$ such that

$$
\left\|u_{i}(\cdot, t)\right\|_{\infty} \leq l\left[\beta \sup _{t \geq t_{0}}\|\mathbf{u}(\cdot, t)\|_{\infty}+A+\epsilon\right](1+\epsilon) \quad \text { for all } t \geq t_{1} \text { and } i=1, \ldots, N .
$$

Thus, we have

$$
\sup _{t \geq t_{1}}\|\mathbf{u}(\cdot, t)\|_{\infty} \leq l\left[\beta \sup _{t \geq t_{0}}\|\mathbf{u}(\cdot, t)\|_{\infty}+A+\epsilon\right](1+\epsilon)
$$

Note that, by (9), we have

$$
\left|\frac{\partial u_{i}(x, t)}{\partial t}+\frac{1}{l} u_{i}(x, t)\right| \leq \beta \sup _{t \geq t_{1}}\|\mathbf{u}(\cdot, t)\|_{\infty}+A+\epsilon
$$


in $D_{t_{1}+r}$ for $i=1, \ldots, N$. Plugging (13) into (14), we obtain

$$
\left|\frac{\partial u_{i}(x, t)}{\partial t}+\frac{1}{l} u_{i}(x, t)\right| \leq l\left[\beta^{2} \sup _{t \geq t_{0}}\|\mathbf{u}(\cdot, t)\|_{\infty}+\beta(A+\epsilon)\right](1+\epsilon)+A+\epsilon,
$$

in $D_{t_{1}+r}$ for each $i=1, . ., N$.

Again, by Lemma 3.1, there exists a $t_{2}>t_{1}$ such that

$$
\left\|u_{i}(\cdot, t)\right\|_{\infty} \leq\left[l^{2} \beta^{2} \sup _{t \geq t_{0}}\|\mathbf{u}(\cdot, t)\|_{\infty}+l^{2} \beta(A+\epsilon)\right](1+\epsilon)^{2}+l(A+\epsilon)(1+\epsilon)
$$

for all $t \geq t_{2}$ and $i=1, \ldots, N$. Repeating the above process, one can find a sequence $t_{k} \uparrow \infty$ such that for all $k \in \mathbf{N}$,

$$
\sup _{t \geq t_{k}}\|\mathbf{u}(\cdot, t)\|_{\infty} \leq R_{\epsilon}^{k} \sup _{t \geq t_{0}}\|\mathbf{u}(\cdot, t)\|_{\infty}+l(A+\epsilon)(1+\epsilon)\left(1+R_{\epsilon}+\ldots+R_{\epsilon}^{k-1}\right),
$$

where $R_{\epsilon}:=l \beta(1+\epsilon) \in(0,1)$. Since $R_{\epsilon} \in(0,1)$, we have

$$
\lim _{k \rightarrow \infty}\left[\sup _{t \geq t_{k}}\|\mathbf{u}(\cdot, t)\|_{\infty}\right] \leq \frac{l(A+\epsilon)(1+\epsilon)}{1-R_{\epsilon}} .
$$

As $\epsilon>0$ is arbitrary, we have justified (10) and the proof is completed.

\section{Absolute stability and synchronization}

We shall discuss absolute stability and synchronization for system (3) in Subsections 4.1 and 4.2 respectively.

\subsection{Absolute stability}

For system (3) with a fixed input $\mathbf{I}(x, t)$, starting from an arbitrary initial value $\mathbf{V}_{0}$, the solution $\mathbf{V}(x, t)$ exists for all $t \geq 0$, by Theorem 2.1. $\mathbf{V}(x, t)$ is said to be absolutely stable if

(i) the solution $\mathbf{U}(x, t)$ of (3) evolved from any initial value close to $\mathbf{V}_{0}$ remains close to $\mathbf{V}(x, t)$ for all $t \geq 0$, and

(ii) $\mathbf{U}(x, t)$ approaches $\mathbf{V}(x, t)$ as $t \rightarrow \infty$ uniformly for $x \in \Omega$ for any solution $\mathbf{U}(x, t)$ of (3).

We say that a system is absolutely stable if all its solutions are absolutely stable. The notion of absolute stability was introduced in [13] to depict a dynamical element in neuronal systems: the activities forget their initial states but do not forget their inputs.

Previous work [13] employed the Lyapunov functional approach to derive a sufficient condition for the absolute stability of system (3) when time delay is not taken into account, i.e., $\tau \equiv \mathbf{0}$. Asymptotic stability of the origin for the linearized (3) at a stationary solution when the external input $\mathbf{I}$ is time-independent, has been reported in [12]. Absolute stability for the neural field model (3) with propagation time delays has not been reported, to the best of our knowledge. Here we shall provide a criterion for the absolute stability in the delay model (3). Furthermore, our approach is also valid for unbounded $\Omega$. To present our approach, we first replace (A3) and (A4) by the following conditions:

(A3') $\mathbf{W} \in B C\left(\left[-\tau^{M}, \infty\right) ; \mathcal{Y}_{\alpha}\right)$,

$\left(\mathrm{A}^{\prime}{ }^{\prime}\right) \quad \mathbf{I} \in B C\left(\left[-\tau^{M}, \infty\right) ; \mathcal{X}\right)$.

For convenience, we lump conditions (A1), (A2), (A3') and (A4') together as condition (H). In this section, we always assume that $(\mathrm{H})$ holds. Let us state the main result of this paper:

Theorem 4.1. The system (3) is absolutely stable provided

$$
l_{\max } W_{\infty}\left\|\mathbf{S}^{\prime}\right\|_{\infty}<1,
$$

where $l_{\max }:=\max _{i=1, \ldots, N} l_{i}$ and $W_{\infty}:=\sup _{x \in \Omega, t>0}\|\mathbf{W}(x, \cdot, t)\|_{L^{1}(\Omega)}$. 
Remark 4.1. We remark that our sufficient condition (16) for absolute stability is similar to the one in [13, Theorem 4.7] (without time delays), which is expressed by

$$
l_{\max }\|g\|_{\mathcal{G}}\left\|\mathbf{S}^{\prime}\right\|_{\infty}<1
$$

where the functional $g$ is defined by $g(S)(x, t):=\int_{\Omega} W(x, y, t) S(y)$ dy for $S \in C(\Omega)$ and $\mathcal{G}$ is the pre-Hilbert space (with the usual inner product) defined on $C(\Omega)$. It seems not straightforward to compare these two bounds. However, if the connectivity matrix $\mathbf{W}$ is translation invariant, (17) can be reduced to calculating the eigenvalue of some Hermitian matrix, see [13, Theorem 4.9] and [13, p.231]. Then it is possible to compare (16) and (17). In fact, the two bounds can be better to each other depending on the choice of $\mathbf{W}$. More details are presented in Section 5 . We stress that the present Theorem 4.1 applies to the delay case.

In general, the dynamics of system (3) can be very complicated, depending on how $\mathbf{W}$ and $\mathbf{I}$ are chosen. When both $\mathbf{W}$ and $\mathbf{I}$ are time-independent, i.e.,

$$
\frac{\partial \mathbf{V}(x, t)}{\partial t}=-\mathbf{L V}(x, t)+\int_{\Omega} \mathbf{W}(x, y) \mathbf{S}(\mathbf{V}(y, t-\tau(x, y))) d y+\mathbf{I}(x),
$$

the dynamics can be investigated by analyzing the stability of stationary solutions (if they exist). In fact, Theorem 4.1 shows that, under condition (16), if stationary solutions exist, it must be unique and globally asymptotically stable, which means that the global dynamics is quite simple.

In the next result, we shall show the existence of stationary solutions to system (18). Then its uniqueness and global stability are a consequence of Theorem 4.1 .

Theorem 4.2. Let $\Omega$ be either the whole space $\mathbf{R}^{n}$ or a compact subset of $\mathbf{R}^{n}$. Furthermore, if $\Omega=\mathbf{R}^{n}$, we assume that

$$
\lim _{\|x\| \rightarrow \infty} \sum_{j=1}^{N} \int_{\mathbf{R}^{n}}\left|W_{i j}(x, y)\right| d y=0 \text { and } \lim _{\|x\| \rightarrow \infty} I_{i}(x)=0, i=1, . ., N .
$$

Then system (18) has a stationary solution. Furthermore, the solution is unique and is globally asymptotically stable as long as condition (16) holds.

Remark 4.2. (i) The existence of stationary solution in $L^{2}(\Omega)$, with compact and connected $\Omega$, for system (18) was established in [11]. (ii) Our sufficient condition for the stability of stationary solutions, (16), can be rewritten as

$$
l_{\max }\left[\sup _{x \in \Omega} \max _{i=1, \ldots, N} \sum_{j=1}^{N} \int_{\Omega}\left|W_{i j}(x, y)\right| d y\right]\left\|\mathbf{S}^{\prime}\right\|_{\infty}<1 .
$$

On the other hand, a sufficient condition for local stability of stationary solutions of (18) has been reported in [12, Theorem 4.2.3], which reads as

$$
\int_{\Omega}\left[\sum_{i, j=1}^{N} l_{i}^{2} \int_{\Omega}\left|\widetilde{W}_{i j}(x, y)\right|^{2} d y\right] d x<1
$$

where $\widetilde{W}_{i j}=(\widetilde{\boldsymbol{W}})_{i, j}$ and $\widetilde{\boldsymbol{W}}(x, y):=\mathbf{W}(x, y) D \mathbf{S}\left(V^{0}(y)\right)$. Here $V^{0}$ is a stationary solution of (18). Condition (20) involves a $L^{1}$ norm on $W_{i j}$, whereas (21) includes a $L^{2}$-norm on $\widetilde{W}$. In general, it is nontrivial to compare (20) with (21) since (21) depends on the value of $V^{0}$, which is usually unavailable or implicit. In some computable cases, it can be shown that (20) is weaker than (21). (iii) Via analyzing the spectrum of the infinitesimal generator associated with the linearized system, a more complicated criterion for asymptotical stability of the origin was established in [36]. In this regard, the choice of the function space is again crucial for the validity of linearized stability analysis. With $\mathcal{X}=C(\bar{\Omega})$, the spectral properties for the generator of the semigroup associated with the linearized system at a steady state were analyzed in [32]. We also note that a sufficient condition for stability of stationary solutions obtained by estimating the eigenvalues of some self-adjoint operator arising from system (18) without time delay was reported in [11]. 
The rest of this subsection is devoted to proving Theorems 4.1 and 4.2. First, we need some preparations.

Lemma 4.1. Let $I_{\infty}:=\sup _{t>0}\|\mathbf{I}(\cdot, t)\|_{\infty}$. Then for $i=1, \ldots, N$,

$$
\sup _{t \geq 0}\left\|V_{i}(\cdot, t)\right\|_{\infty} \leq K_{\infty}:=\max \left\{\left\|\mathbf{V}_{0}\right\|_{C},\left(W_{\infty}\|\mathbf{S}\|_{\infty}+I_{\infty}\right) l_{\max }\right\}
$$

Proof. Notice that $I_{\infty}<\infty$ due to (A4'). Set $M:=W_{\infty}\|\mathbf{S}\|_{\infty}+I_{\infty}$. For each $i=1, \ldots, N$, with (H), it follows from (1) that

$$
\left|\frac{\partial V_{i}(x, t)}{\partial t}+\frac{1}{l_{i}} V_{i}(x, t)\right| \leq M, \quad \text { for all } x \in \Omega, t \geq 0 .
$$

By comparing with the following ODE

$$
u^{\prime}(t)=-\frac{1}{l_{\max }} u(t)+M, \quad t>0, \quad u(0)=\left\|\mathbf{V}_{0}\right\|_{C},
$$

it follows that

$$
\sup _{t \geq 0}\left\|V_{i}(\cdot, t)\right\|_{\infty} \leq \max \left\{\left\|\mathbf{V}_{0}\right\|_{C}, M l_{\max }\right\}
$$

for $i=1, \ldots, N$ and all $t \geq \tilde{T}$ for some $\tilde{T}$. This completes the proof.

Proposition 4.1. If (16) holds, then the difference of any two solutions of (3) tends to zero as $t \rightarrow \infty$ regardless of their initial data.

Proof. Let $\mathbf{U}$ and $\mathbf{V}$ be two solutions of (3) evolved from any two initial values $\phi^{U}$ and $\phi^{V}$. We introduce their difference $\mathbf{Z}:=\mathbf{U}-\mathbf{V}$ with $\mathbf{Z}=\left(Z_{1}, \ldots, Z_{N}\right)$. We shall show that $\left\|Z_{i}(\cdot, t)\right\|_{\infty} \rightarrow 0$ as $t \rightarrow \infty$ for $i=1, \ldots, N$. From (1), we see that

$$
\begin{aligned}
\frac{\partial Z_{i}(x, t)}{\partial t}= & -\frac{1}{l_{i}} Z_{i}(x, t) \\
& +\sum_{j=1}^{N} \int_{\Omega} W_{i j}(x, y, t)\left[S_{j}\left(U_{j}\left(y, t-\tau_{j}(x, y)\right)\right)-S_{j}\left(V_{j}\left(y, t-\tau_{j}(x, y)\right)\right)\right] d y
\end{aligned}
$$

for $i=1, \ldots, N$. Applying the mean value theorem yields

$$
\begin{aligned}
\left|\frac{\partial Z_{i}(x, t)}{\partial t}+\frac{1}{l_{i}} Z_{i}(x, t)\right| & \leq\left\|\mathbf{S}^{\prime}\right\|_{\infty} \sum_{j=1}^{N} \int_{\Omega}\left|W_{i j}(x, y, t) \| Z_{j}\left(y, t-\tau_{j}(x, y)\right)\right| d y \\
& \leq\left\|\mathbf{S}^{\prime}\right\|_{\infty} W_{\infty} \sup _{t \geq s}\|\mathbf{Z}(\cdot, t)\|_{\infty},
\end{aligned}
$$

in $D_{s+\tau^{M}}$ for all $s \geq 0$, where $D_{s}:=\{(x, t): x \in \Omega, t \geq s\}$.

Note that $\mathbf{Z} \in C^{0,1}\left(D_{0} ; \mathbf{R}^{N}\right)$. Also, by Lemma 4.1, we obtain

$$
\left|Z_{i}(x, t)\right| \leq 2 K_{\infty} \quad \text { for all }(x, t) \in D_{0} \text { and } i=1, \ldots, N .
$$

Together with (22) and (16), we can apply Lemma 3.2 with $\beta=\left\|\mathbf{S}^{\prime}\right\|_{\infty} W_{\infty}, l=l_{\max }$ and $A=0$ to conclude that $\left\|Z_{i}(\cdot, t)\right\|_{\infty} \rightarrow 0$ as $t \rightarrow \infty$ for $i=1, \ldots, N$. The proof is completed.

Let us justify Theorem 4.1 .

Proof of Theorem 4.1: Due to Proposition 4.1, it suffices to show that all solutions are stable; namely, for any given $\epsilon>0$ and a solution $\mathbf{V}$ with initial data $\phi^{V}$, there exists a $\delta>0$ such that if a solution $\mathbf{U}$ with initial data $\phi^{U}$ satisfying $\sup _{\theta \in\left[-\tau^{M}, 0\right]}\left\|\phi^{V}(\theta)-\phi^{U}(\theta)\right\|_{\infty}<\delta$, then

$$
\|\mathbf{U}(\cdot, t)-\mathbf{V}(\cdot, t)\|_{\infty}<\epsilon \quad \text { for all } t \geq 0 .
$$


Set $\mathbf{Z}:=\mathbf{U}-\mathbf{V}$. First, we choose $T>\tau^{M}$. For given $\epsilon>0$, using Proposition 2.1 we choose a small $\delta>0$ such that

$$
\|\mathbf{Z}(\cdot, t)\|_{\infty}<\epsilon, \quad t \in[0, T]
$$

whenever $\sup _{\theta \in\left[-\tau^{M}, 0\right]}\left\|\phi^{V}(\theta)-\phi^{U}(\theta)\right\|_{\infty}<\delta$.

We now show that (23) actually holds for all $t \in[0, \infty)$. Indeed, for each $x \in \Omega$ and $t \geq T$, it follows from (22) that

$$
\left|\frac{\partial Z_{i}(x, t)}{\partial t}+\frac{1}{l_{i}} Z_{i}(x, t)\right| \leq \epsilon\left\|\mathbf{S}^{\prime}\right\|_{\infty} W_{\infty}=: b
$$

as long as $\|\mathbf{Z}(\cdot, s)\|_{\infty}<\epsilon$ for all $s \in\left[t-\tau^{M}, t\right]$. As in the proof of Lemma 3.1, one observes

$$
\frac{\partial Z_{i}(x, t)}{\partial t}>0 \quad \text { if } Z_{i}(x, t)<-l_{i} b, \quad \frac{\partial Z_{i}(x, t)}{\partial t}<0 \quad \text { if } Z_{i}(x, t)>l_{i} b
$$

as long as $\|\mathbf{Z}(\cdot, s)\|_{\infty}<\epsilon$ for all $s \in\left[t-\tau^{M}, t\right]$. Note that the condition (16) yields $\left[-l_{i} b, l_{i} b\right] \subset[-\epsilon, \epsilon]$ for each $i=1, \ldots, N$. Together with (23) and (24), we can easily conclude that $\mathbf{Z}(x, t)$ always stays in $[-\epsilon, \epsilon]^{N}$ for all $x \in \Omega$ and $t \geq T$. Thus, all solutions of (1) are stable and this completes the proof of Theorem 4.1 .

Proof of Theorem 4.2: We first show that (18) admits a stationary solution, i.e., there exists $\mathbf{V}_{*}=\mathbf{V}_{*}(x)$ satisfying

$$
0=-\mathbf{L} \mathbf{V}_{*}(x)+\int_{\Omega} \mathbf{W}(x, y) \mathbf{S}\left(\mathbf{V}_{*}(y)\right) d y+\mathbf{I}(x) .
$$

For this, we define the operator $\mathcal{F}: B C(\Omega) \rightarrow B C(\Omega)$ by

$$
\mathcal{F} \mathbf{u}=\mathbf{L}^{-1} \int_{\Omega} \mathbf{W}(x, y) \mathbf{S}(\mathbf{u}(y)) d y+\mathbf{L}^{-1} \mathbf{I}(x)
$$

Then it suffices to show that $\mathcal{F}: B C(\Omega) \rightarrow B C(\Omega)$ has a fixed point (under the supremum norm). Note that if $\Omega$ is compact, $B C(\Omega) \equiv C(\Omega)$.

Choosing a closed convex set

$$
D:=\left\{\mathbf{u} \in B C(\Omega):\|\mathbf{u}\|_{\infty} \leq K\right\},
$$

where $K:=l_{\max }\|\mathbf{S}\|_{\infty}\|\mathbf{W}\|_{y_{\alpha}}+l_{\max } I_{\infty}$ and $l_{\max }$ is defined in (16), then we can easily obtain $\mathcal{F}(D) \subset D$.

To apply the Schauder's fixed point theorem, it suffices to show that $\mathcal{F}$ is continuous on $D$ and $\mathcal{F}(D)$ is a relatively compact subset of $D$. It follows from the assumption $(\mathrm{H})$ that

$$
\|\mathcal{F}(\mathbf{u})-\mathcal{F}(\mathbf{v})\|_{\infty} \leq l_{\max }\left\|\mathbf{S}^{\prime}\right\|_{\infty}\|\mathbf{W}\|_{y_{\alpha}}\|\mathbf{u}-\mathbf{v}\|_{\infty}, \text { for any } \mathbf{u}, \mathbf{v} \in D
$$

which implies the continuity of $\mathcal{F}$.

We now justify the relative compactness of $\mathcal{F}(D)$. For this part, we shall divide our discussion into two cases: (i) $\Omega$ is a compact subset of $\mathbf{R}^{n}$, and (ii) $\Omega=\mathbf{R}^{n}$. For (i), using (H) we have

$$
\left|\mathcal{F}_{i}(\mathbf{u})(x)-\mathcal{F}_{i}(\mathbf{u})(\hat{x})\right| \leq l_{\max }\left[\|\mathbf{S}\|_{\infty}\|\mathbf{W}\|_{y_{\alpha}}\|x-\hat{x}\|^{\alpha}+\left|I_{i}(x)-I_{i}(\hat{x})\right|\right]
$$

for all $i=1, \ldots, N, x, \hat{x} \in \Omega$ and $\mathbf{u} \in D$, where $\mathcal{F}(\mathbf{u}):=\left(\mathcal{F}_{1}(\mathbf{u}), \ldots, \mathcal{F}_{N}(\mathbf{u})\right)$. Note that the compactness of $\Omega$ implies the uniform continuity of $I_{i}$. Hence we see from $(26)$ that $\mathcal{F}(D)$ is equicontinuous. Also, note that $\mathcal{F}(D)$ is uniformly bounded since $\|\mathcal{F}(\mathbf{u})\|_{\infty} \leq K$. By the Arzela-Ascoli theorem, we obtain the relative compactness of $\mathcal{F}(D)$.

For (ii), following the same process as in (i), we have the equicontinuity and uniform boundedness of $\mathcal{F}(D)$ when $\Omega=\mathbf{R}^{n}$ (the uniform continuity of $I_{i}$ over $\mathbf{R}^{n}$ follows from the assumption that $\lim _{\|x\| \rightarrow \infty} I_{i}(x)$ exists). However, the Arzela-Ascoli theorem cannot be applied to guarantee the relative compactness of $\mathcal{F}(D)$ since $\mathbf{R}^{n}$ is not compact. In fact, if all functions in $\mathcal{F}(D)$ tend to zero uniformly at infinity, i.e., for each $\varepsilon>0$, there exists a $L>0$ such that

$$
\left|\mathcal{F}_{i}(\mathbf{u})(x)\right|<\varepsilon \text { for all } i=1, \ldots, N, \mathbf{u} \in D \text { and }\|x\| \geq L,
$$


then the Arzela-Ascoli theorem can be generalized to $\Omega=\mathbf{R}^{n}$ (see, for example, [19, P.46-47]). For this, observe that

$$
\left|\mathcal{F}_{i}(\mathbf{u})(x)\right| \leq l_{\max }|| \mathbf{S} \|_{\infty} \sum_{j=1}^{N} \int_{\mathbf{R}^{n}}\left|W_{i j}(x, y)\right| d y+l_{\max }\left|I_{i}(x)\right|, i=1, \ldots, N, \mathbf{u} \in D .
$$

By assumption (19), we then obtain (27). Thus the relative compactness of $\mathcal{F}(D)$ is confirmed.

Consequently, the Schauder's fixed point theorem yields that there exists a solution to (25). When (16) holds, the uniqueness and the globally asymptotical stability of the solution follow from Theorem 4.1.

\subsection{Synchronization}

In this subsection, we shall investigate the synchronizations for system (3). More precisely, we shall consider two types of synchronization:

$$
\begin{aligned}
& \max _{i, j \in\{1,2, \ldots, N\}}\left\|V_{i}(\cdot, t)-V_{j}(\cdot, t)\right\|_{\infty} \rightarrow 0 \quad \text { as } t \rightarrow \infty ; \\
& \sup _{x, \bar{x} \in \Omega}\left|V_{i}(x, t)-V_{i}(\bar{x}, t)\right| \rightarrow 0 \quad \text { as } t \rightarrow \infty, i=1, \ldots, N .
\end{aligned}
$$

Notice that (28) describes the phenomenon that different layers (or populations) of neurons synchronize, whereas (29) describes the phenomenon that the system synchronizes within each layer. We shall give some basic criteria for (28) and (29) to take place, respectively.

To establish the synchronization for (3) among different layers, we shall try to estimate $\left\|V_{i}(\cdot, t)-V_{i+1}(\cdot, t)\right\|_{\infty}$. Let us fix $x, y, t$ and denote the $i$ th row sum of $\mathbf{W}=\mathbf{W}(x, y, t)$ as $\rho_{i}=\rho_{i}(x, y, t):=\sum_{j=1}^{N} W_{i j}(x, y, t)$. We compose a matrix $\tilde{\mathbf{W}}$ whose entries comprise $W_{i j}$ and row sum $\rho_{i}$ of $\mathbf{W}$ :

$$
\tilde{\mathbf{W}}=\left[\tilde{W}_{i j}\right]_{1 \leq i, j \leq N} \in \mathbf{R}^{N \times N},
$$

where

$$
\tilde{W}_{i j}:= \begin{cases}W_{i i}-\rho_{i} & \text { if } i=j, \\ W_{i j} & \text { otherwise. }\end{cases}
$$

From $\tilde{\mathbf{W}}$, we further construct a matrix $\hat{\mathbf{W}}$ :

$$
\hat{\mathbf{W}}=\left[\hat{W}_{i j}\right]_{1 \leq i, j \leq N-1}:=\mathbf{C} \tilde{\mathbf{W}} \mathbf{C}^{T}\left(\mathbf{C} \mathbf{C}^{T}\right)^{-1} \in \mathbf{R}^{(N-1) \times(N-1)},
$$

where $T$ denotes transpose, and $\mathbf{C}$ is the following $(N-1) \times N$ matrix

$$
\mathbf{C}:=\left(\begin{array}{ccccc}
1 & -1 & 0 & \cdots & 0 \\
0 & 1 & -1 & \ddots & \vdots \\
\vdots & \ddots & \ddots & \ddots & 0 \\
0 & \cdots & 0 & 1 & -1
\end{array}\right) .
$$

It can be shown that $\hat{\mathbf{W}}$ is well defined and satisfies

$$
\mathbf{C} \tilde{\mathbf{W}}=\hat{\mathbf{W}} \mathbf{C},
$$

by arguments similar to those in the appendix of [26]. Thus $\mathbf{C} \tilde{\mathbf{W}}\left(\xi_{1}, \ldots, \xi_{N}\right)^{T}=\hat{\mathbf{W}} \mathbf{C}\left(\xi_{1}, \ldots, \xi_{N}\right)^{T}$, i.e.,

$$
\sum_{j=1}^{N}\left[\tilde{W}_{i j}-\tilde{W}_{i+1, j}\right] \xi_{j}=\sum_{j=1}^{N-1} \hat{W}_{i j}\left(\xi_{j}-\xi_{j+1}\right),
$$

for $\left(\xi_{1}, \ldots, \xi_{N}\right) \in \mathbf{R}^{N}$. This process can be regarded as a rearrangement with a transformation for the terms in the summation. 
Theorem 4.3. Under the following assumptions

(i) $l_{i}=l_{j}=: l, \tau_{i}(x, y)=\tau_{j}(x, y)=: \theta(x, y)$ and $S_{i}(x)=S_{j}(x)=: S(x)$ for all $i, j=1, \ldots, N$,

(ii) $\max _{i, j \in\{1, \ldots, N\}} \sup _{x \in \Omega}\left\|\rho_{i}(x, \cdot, t)-\rho_{j}(x, \cdot, t)\right\|_{L^{1}(\Omega)} \rightarrow 0$ as $t \rightarrow \infty$,

(iii) $\omega(t):=\max _{i, j \in\{1, \ldots, N\}}\left\|I_{i}(\cdot, t)-I_{j}(\cdot, t)\right\|_{\infty} \rightarrow 0$ as $t \rightarrow \infty$,

the synchronization for (3) among different layers takes place (i.e., (28) holds) as long as condition

$$
l_{\max }\left(W_{\infty}+\hat{W}_{\infty}\right)\left\|\mathbf{S}^{\prime}\right\|_{\infty}<1
$$

holds, where $\hat{W}_{\infty}:=\sup _{x \in \Omega, t>0}\|\hat{\mathbf{W}}(x, \cdot, t)\|_{L^{1}(\Omega)}$ and $W_{\infty}:=\sup _{x \in \Omega, t>0}\|\mathbf{W}(x, \cdot, t)\|_{L^{1}(\Omega)}$.

Proof. Let $\mathbf{V}=\left(V_{1}, \ldots, V_{N}\right)^{T}$ be a solution of (3). Set $Z_{i}(x, t)=V_{i}(x, t)-V_{i+1}(x, t)(\bmod N)$ for $i=1, \ldots, N$. Then by assumption (i), we have

$$
\begin{aligned}
\frac{\partial Z_{i}(x, t)}{\partial t}+\frac{1}{l} Z_{i}(x, t)= & \sum_{j=1}^{N} \int_{\Omega} W_{i j}(x, y, t) S\left(V_{j}(y, t-\theta(x, y))\right) d y+I_{i}(x, t) \\
& -\sum_{j=1}^{N} \int_{\Omega} W_{i+1, j}(x, y, t) S\left(V_{j}(y, t-\theta(x, y))\right) d y-I_{i+1}(x, t),
\end{aligned}
$$

for $i=1, \ldots, N-1$. For the terms in the summations in (32),

$$
\begin{aligned}
& \sum_{j=1}^{N}\left[W_{i j}(x, y, t)-W_{i+1, j}(x, y, t)\right] S\left(V_{j}(y, t-\theta(x, y))\right) \\
= & \rho_{i}(x, y, t) S\left(V_{i}(y, t-\theta(x, y))\right)-\rho_{i+1}(x, y, t) S\left(V_{i+1}(y, t-\theta(x, y))\right) \\
& +\sum_{j=1}^{N}\left[\tilde{W}_{i j}(x, y, t)-\tilde{W}_{i+1, j}(x, y, t)\right] S\left(V_{j}(y, t-\theta(x, y))\right) \\
=\quad & \rho_{i}(x, y, t) S\left(V_{i}(y, t-\theta(x, y))\right)-\rho_{i+1}(x, y, t) S\left(V_{i+1}(y, t-\theta(x, y))\right) \\
& +\sum_{j=1}^{N-1} \hat{W}_{i j}(x, y, t)\left[S\left(V_{j}(y, t-\theta(x, y))\right)-S\left(V_{j+1}(y, t-\theta(x, y))\right)\right],
\end{aligned}
$$

by (30). Thus,

$$
\begin{aligned}
& \left|\frac{\partial Z_{i}(x, t)}{\partial t}+\frac{1}{l} Z_{i}(x, t)\right| \\
\leq & \int_{\Omega}\left|\rho_{i}(x, y, t) S\left(V_{i}(y, t-\theta(x, y))\right)-\rho_{i+1}(x, y, t) S\left(V_{i+1}(y, t-\theta(x, y))\right)\right| d y \\
& +\sum_{j=1}^{N-1} \int_{\Omega}\left|\hat{W}_{i j}(x, y, t)\right|\left|S\left(V_{j}(y, t-\theta(x, y))\right)-S\left(V_{j+1}(y, t-\theta(x, y))\right)\right| d y+\omega(t) \\
\leq & \int_{\Omega}\left|\rho_{i}(x, y, t)\right|\left|S\left(V_{i}(y, t-\theta(x, y))\right)-S\left(V_{i+1}(y, t-\theta(x, y))\right)\right| d y \\
& +\int_{\Omega}\left|\rho_{i}(x, y, t)-\rho_{i+1}(x, y, t) \| S\left(V_{i+1}(y, t-\theta(x, y))\right)\right| d y \\
& +\sum_{j=1}^{N-1} \int_{\Omega}\left|\hat{W}_{i j}(x, y, t) \| S\left(V_{j}(y, t-\theta(x, y))\right)-S\left(V_{j+1}(y, t-\theta(x, y))\right)\right| d y+\omega(t) .
\end{aligned}
$$


Note that

$$
\int_{\Omega}\left|\rho_{i}(x, y, t)\right| d y \leq \sum_{j=1}^{N} \int_{\Omega}\left|W_{i j}(x, y, t)\right| d y \leq W_{\infty} .
$$

By the mean value theorem, we obtain

$$
\begin{aligned}
& \left|\frac{\partial Z_{i}(x, t)}{\partial t}+\frac{1}{l} Z_{i}(x, t)\right| \\
\leq & \left\|\mathbf{S}^{\prime}\right\|_{\infty}\left(W_{\infty}+\hat{W}_{\infty}\right) \sup _{t \geq s}\|\mathbf{Z}(\cdot, t)\|_{\infty}+\|\mathbf{S}\|_{\infty}\left\|\rho_{i}(x, \cdot, t)-\rho_{i+1}(x, \cdot, t)\right\|_{L^{1}(\Omega)}+\omega(t)
\end{aligned}
$$

in $D_{s+\tau^{M}}$, for all $s \geq 0$ and $i=1, \ldots, N$, where $D_{s}:=\{(x, t): x \in \Omega, t \geq s\}$. By letting $\beta=\left\|\mathbf{S}^{\prime}\right\|_{\infty}\left(W_{\infty}+\hat{W}_{\infty}\right)$, we see that (31) implies $l \beta<1$. Also, note that $\left|Z_{i}(x, t)\right| \leq 2 K_{\infty}$ for all $(x, t) \in D_{0}$ and $i=1, \ldots, N$ (by Lemma 4.1). Then by (ii) and (iii), we can use Lemma 3.2 to conclude that

$$
\left\|Z_{i}(\cdot, t)\right\|_{\infty} \rightarrow 0 \quad \text { as } t \rightarrow \infty \text { for each } i=1, \ldots, N .
$$

The proof is completed.

We note that the estimation in (34) can be relaxed by imposing a condition on row sums $\rho_{i}$ or we can lump up the first and the last integrals in (33) into one summation and define a new matrix in terms of $\hat{W}_{i j}$ and $\rho_{i}$, and then impose a condition on this matrix. These will weaken condition (31).

An $N \times N$ matrix $\mathbf{B}=\left[b_{i j}\right]_{1 \leq i, j \leq N}$ is called "circulant" [8] if $\left[b_{i j}\right]_{1 \leq i, j \leq N}=\operatorname{circ}\left(b_{1}, \ldots, b_{N}\right)$ for some $b_{k}, k=1, \ldots, N$, i.e., each row of $\mathbf{B}$ is a right cyclic shift of the row above it. Obviously, a circulant matrix has identical row sums. If matrix $\mathbf{W}$ has identical row sums, then condition (ii) in Theorem 4.3 can be lifted. Moreover, if $\mathbf{W}$ is circulant, then (31) can be replaced by condition (16).

Corollary 4.1. System (3) attains synchronization among different layers under assumptions (i) and (iii) of Theorem 4.3, and condition (31) if $\mathbf{W}$ has identical row sums, and condition (16) if $\mathbf{W}$ is circulant.

Proof. If $\mathbf{W}$ has identical row sums, then assumption (ii) of Theorem 4.3 holds obviously. If $\mathbf{W}$ is circulant, we have $W_{i+1, j}=W_{i, j-1}(\bmod N)$. Set $Z_{i}(x, t)=V_{i}(x, t)-V_{i+1}(x, t)(\bmod N)$ for $i=1, \ldots, N$. Then $(32)$ and $(35)$ reduce to

$$
\begin{aligned}
\left|\frac{\partial Z_{i}(x, t)}{\partial t}+\frac{1}{l_{i}} Z_{i}(x, t)\right| & \leq\left\|\mathbf{S}^{\prime}\right\|_{\infty} \sum_{j=1}^{N} \int_{\Omega}\left|W_{i j}(x, y, t) \| Z_{j}(y, t-\theta(x, y))\right| d y \\
& \leq\left\|\mathbf{S}^{\prime}\right\|_{\infty} W_{\infty} \sup _{t \geq s}\|\mathbf{Z}(\cdot, t)\|_{\infty}
\end{aligned}
$$

in $D_{s+\tau^{M}}$ for all $s \geq 0$, where $D_{s}:=\{(x, t): x \in \Omega, t \geq s\}$. As in the proof of Theorem 4.3, we see that the assertion holds under condition (16). The proof is completed.

Remark 4.3. Assumption (ii) in Theorem 4.3 somehow depicts a sense of (eventual) balance of coupling weights among all layers, and hence the synchronization among different layers becomes possible. Circulant coupling and diffusive coupling: $\rho_{i}(x, y, t) \equiv 0$ for all $i$, are conditions commonly imposed on the connection matrix in the study of coupled network systems. They indicate corresponding network structures in the systems. What we have discussed in this subsection is about identical synchronization (or perfect synchronization), which is a idealized notion. More practical consideration should be approximate synchronization which allows a synchronization error as $t \rightarrow \infty$, i.e., the limits to zeros in (28) and (29) being replaced by a small bound $\varepsilon>0$, cf. [17]. With such a notion, assumptions (i) and (ii) in Theorem 4.3 can all be relaxed. In fact, the difference between row sums of $\mathbf{W},\left\|\rho_{i}-\rho_{j}\right\|$, and variation of delays, $\left\|\tau_{i}-\tau_{j}\right\|$, all contribute to the synchronization error $\varepsilon$.

The following theorem is for synchronization within each layer. 
Theorem 4.4. Assume that $\Omega=\mathbf{R}^{n}$ and

(i) $\mathbf{W}(x, y, t)=\mathbf{W}(x-y)$ for all $x, y \in \mathbf{R}^{n}$ and $t \geq 0$,

(ii) $\mathbf{I}(x, t)=\mathbf{I}^{*}$ for some $\mathbf{I}^{*} \in \mathbf{R}^{N}$, for all $x \in \mathbf{R}^{n}$ and $t \geq 0$.

Then all solutions of (3) converge to a trivial solution (constant in space and time) as long as (16) holds. In particular, (29) holds and the system synchronizes within each layer.

Proof. Set the operator $\mathcal{F}: \mathbf{R}^{N} \rightarrow \mathbf{R}^{N}$ by $\mathcal{F} \mathbf{u}=\mathbf{L}^{-1} \int_{\mathbf{R}^{n}} \mathbf{W}(x-y) \mathbf{S}(\mathbf{u}) d y+\mathbf{L}^{-1} \mathbf{I}^{*}$, where $\mathbf{u}=\left(u_{1}, . ., u_{N}\right)$ is a constant vector in $\mathbf{R}^{N}$. Then (3) has a trivial solution if and only if $\mathcal{F}$ has a fixed point in $\mathbf{R}^{N}$. Let $z=x-y$. For each $i=1, \ldots, N$,

$$
\sum_{j=1}^{N} \int_{\mathbf{R}^{n}} W_{i j}(x-y) S_{j}\left(u_{j}\right) d y=\sum_{j=1}^{N} S_{j}\left(u_{j}\right) \int_{\mathbf{R}^{n}} W_{i j}(z) d z
$$

which implies that $\mathcal{F} \mathbf{u} \in \mathbf{R}^{N}$.

For any $\mathbf{u}$ and $\mathbf{v} \in \mathbf{R}^{N}$, we have

$$
\|\mathcal{F} \mathbf{u}-\mathcal{F} \mathbf{v}\| \leq l_{\max } W_{\infty}\left\|\mathbf{S}^{\prime}\right\|_{\infty}\|\mathbf{u}-\mathbf{v}\|
$$

Due to (16), $\mathcal{F}$ is a contraction. Thus, by the contraction mapping theorem, there exists a unique vector $\mathbf{u}^{*}=$ $\left(u_{1}^{*}, \ldots, u_{N}^{*}\right) \in \mathbf{R}^{N}$ such that $\mathcal{F}\left(\mathbf{u}^{*}\right)=\mathbf{u}^{*}$. In other words, (3) has a trivial solution $\mathbf{u}^{*}$. By Theorem 4.2, $\mathbf{u}^{*}$ is globally asymptotically stable. In particular, given any solution $\mathbf{V}$ of (3), we have

$$
\sup _{x, \bar{x} \in \mathbf{R}^{n}}\left|V_{i}(x, t)-V_{i}(\bar{x}, t)\right| \leq \sup _{x \in \mathbf{R}^{n}}\left|V_{i}(x, t)-u_{i}^{*}\right|+\sup _{\bar{x} \in \mathbf{R}^{n}}\left|V_{i}(\bar{x}, t)-u_{i}^{*}\right|
$$

which tends to zero as $t \rightarrow \infty$ for all $i=1, \ldots, N$. This completes the proof.

We now focus on the homogeneous solutions of system (3). Assume that $\tau$ and $\mathbf{I}$ are space-independent and $\overline{\mathbf{W}}$ does not depend on $x$, where

$$
\overline{\mathbf{W}}=\overline{\mathbf{W}}(t)=\int_{\Omega} \mathbf{W}(x, y, t) d y
$$

then one can consider homogeneous (space-independent) solutions of (3). A homogeneous solution of (3) then satisfies

$$
\dot{\mathbf{V}}(t)=-\mathbf{L V}(t)+\overline{\mathbf{W}}(t) \mathbf{S}(\mathbf{V}(t-\tau))+\mathbf{I}(t), \mathbf{V}(t)=\mathbf{V}_{0}(t), t \in\left[-\tau^{M}, 0\right]
$$

Here $\overline{\mathbf{W}}(t)$ and the external current $\mathbf{I}(t)$ are assumed to be continuous for $t \in[0, \infty)$. Similar to Theorem 2.1, we can obtain the global existence and uniqueness of solution for (37) if $\mathbf{V}_{0} \in C\left(\left[-\tau^{M}, 0\right] ; \mathbf{R}^{N}\right)$. The case with $\tau=0$ and bounded $\Omega$ has been discussed in [13]. By Theorem 4.1, we immediately have the following corollary.

Corollary 4.2. Assume that $\tau$, I, and $\overline{\mathbf{W}}$ in (36) are space-independent, then (29) holds under condition (16).

Proof. By Theorem 4.1, under condition (16), any solution $\overline{\mathbf{V}}(t)$ of (37) must be absolutely stable. Thus every solution of (3) converges uniformly to the homogeneous solution $\overline{\mathbf{V}}(t)$ on $\Omega$. Therefore, for any solution $\mathbf{V}(x, t)$ of (3),

$$
\sup _{x, \bar{x} \in \Omega}\left|V_{i}(x, t)-V_{i}(\bar{x}, t)\right| \leq \sup _{x \in \Omega}\left|V_{i}(x, t)-\bar{V}_{i}(t)\right|+\sup _{\bar{x} \in \Omega}\left|V_{i}(\bar{x}, t)-\bar{V}_{i}(t)\right|
$$

which tends to zero as $t \rightarrow \infty$ for all $i=1, \ldots, N$. This completes the proof. 


\section{Numerical examples}

In this section, we present four numerical examples to illustrate our theoretical results on absolute stability and synchronization in Section 4. Note that in [12], stability theory is established only for stationary solutions of system (3) with time-independent external currents. The numerical examples therein take zero input $(\mathbf{I}=0)$ and illustrate the stability of homogeneous solution $\mathbf{V}=0$. Our Theorem 4.1 concludes absolute stability for system (3) with general input, and thus the numerical simulations herein allow non-constant external currents.

We also design the parameters according to Corollary 4.1 to illustrate synchronization among different layers in system (3), which has not been reported in previous works. We follow the numerical approach used in [12] to solve the system of equations (3). The spatial integration is discretized via the trapezoidal rule and the resulting discretized system of delay ODEs are solved by MATLAB dde23.

We consider system (3) with two layers of neurons $(N=2)$ in one-dimensional spatial domain $(n=1)$ :

$$
\begin{aligned}
& \frac{\partial V_{1}}{\partial t}=-\frac{1}{l} V_{1}+\sum_{j=1}^{2} \int_{0}^{1} \beta_{1 j} e^{-\frac{(x-y)^{2}}{2 \sigma_{1 j}^{2}}} S\left(V_{j}\left(y, t-\frac{|x-y|}{c}\right)\right) d y+I_{1}, \\
& \frac{\partial V_{2}}{\partial t}=-\frac{1}{l} V_{2}+\sum_{j=1}^{2} \int_{0}^{1} \beta_{2 j} e^{-\frac{(x-y)^{2}}{2 \sigma_{2 j}^{2}}} S\left(V_{j}\left(y, t-\frac{|x-y|}{c}\right)\right) d y+I_{2},
\end{aligned}
$$

with initial data

$$
\left(V_{1}(x, t), V_{2}(x, t)\right)=\left(\phi_{1}(x, t), \phi_{2}(x, t)\right), x \in \Omega, t \in\left[-\tau^{M}, 0\right] .
$$

We take the following setting in the numerical examples:

- the space domain $\Omega=[0,1]$;

- the time delays $\tau_{1}(x, y)=\tau_{2}(x, y)=|x-y| / c$ for some $c>0$ so that $\tau^{M}=1 / c$;

- the connectivity matrix $\mathbf{W}=\left[W_{i j}\right]$ with

$$
W_{i j}(x, y, t)=W_{i j}(x-y)=\beta_{i j} e^{-\frac{(x-y)^{2}}{2 \sigma_{i j}^{2}}}, \text { for all } t \geq 0, \quad i, j=1,2,
$$

where

$$
\beta_{i j}:=\frac{\alpha_{i j}}{\sqrt{2 \pi \sigma_{i j}^{2}}}, \quad i, j=1,2 ;
$$

- the activation function $\mathbf{S}(x):=[S(x), S(x)]^{T}$, where $S$ is sigmoidal defined by

$$
S(x):=\frac{1}{1+e^{-x}}-\frac{1}{2} .
$$

Note that the sign of $\alpha_{i j}$ determines whether layer $j$ excites or inhibits layer $i$. It is straightforward to compute that $\left\|\mathbf{S}^{\prime}\right\|_{\infty}=1 / 4$. Herein we choose $l_{1}=l_{2}=: l=4$ (so that $l_{\max }=4$ ) and thus $l_{\max }\left\|\mathbf{S}^{\prime}\right\|_{\infty}=1$. Accordingly, the sufficient condition for absolute stability in Theorem 4.1 becomes

$$
W_{\infty}:=\sup _{x \in[0,1]}\|\mathbf{W}(x, \cdot)\|_{L^{1}([0,1])}<1,
$$

which is equivalent to

$$
\sup _{x \in[0,1]} \max \left\{\sum_{j=1}^{2}\left|\beta_{1 j}\right| \int_{0}^{1} e^{-\frac{(x-y)^{2}}{2 \sigma_{1 j}}} d y, \sum_{j=1}^{2}\left|\beta_{2 j}\right| \int_{0}^{1} e^{-\frac{(x-y)^{2}}{2 \sigma_{2 j}}} d y\right\}<1 .
$$


Example 5.1. We illustrate the absolute stability for system (38)-(39) satisfying condition (40). We take $c=10$ and the external current

$$
\mathbf{I}(x, t):=\left(I_{1}(x, t), I_{2}(x, t)\right), x \in[0,1], t \in[0, \infty),
$$

where $I_{i}$ is a radially symmetric Gaussian, i.e,

$$
I_{i}(x, t):=I_{i}^{*} e^{-(x-1 / 2)^{2} / \kappa_{i}^{2}},
$$

and $I_{1}^{*}=\cos t, I_{2}^{*}=\sin t, \kappa_{i}=1$. In addition, in the connectivity matrix, we choose

$$
\left(\alpha_{11}, \alpha_{12}, \alpha_{21}, \alpha_{22}\right)=(1,2,-4,-3),\left(\sigma_{11}, \sigma_{12}, \sigma_{21}, \sigma_{22}\right)=(2,5,4,6) .
$$

A computation shows that condition (40) is met. Hence the absolute stability of solutions holds and any two solutions approach each other as $t \rightarrow \infty$, regardless of their initial data. For instance, we choose two different initial data:

$$
\begin{aligned}
& \left(\phi_{1}(x, t), \phi_{2}(x, t)\right)=(\sin \pi x, \cos \pi x), x \in[0,1], t \in[-1 / 10,0] \\
& \left(\hat{\phi}_{1}(x, t), \hat{\phi}_{2}(x, t)\right)=\left(e^{-t}-2, e^{t}\right), x \in[0,1], t \in[-1 / 10,0] .
\end{aligned}
$$

Figure 1 indicates that

$$
\|\mathbf{V}(\cdot, t)-\hat{\mathbf{V}}(\cdot, t)\|_{\infty}=\max _{i=1,2} \sup _{x \in[0,1]}\left|V_{i}(x, t)-\hat{V}_{i}(x, t)\right| \rightarrow 0, \text { as } t \rightarrow \infty .
$$

Both solutions appear to converge to a periodic pattern.

Example 5.2. We demonstrate the loss of absolute stability by fixing $\sigma_{i j}>0$, and choosing $\alpha_{i j} \gg 1$ so that (40) fails to hold. For instance, we choose the same data as in Example 5.1 but replace $\alpha_{i j}$ in (41) by

$$
\left(\alpha_{11}, \alpha_{12}, \alpha_{21}, \alpha_{22}\right)=(50,50,-20,-20) \text {. }
$$

Then the absolute stability of solutions is lost, as illustrated in Figure 2.

Example 5.3. To compare with the results in [13], we consider $\tau_{1}=\tau_{2}=0$ (without time delays) in system (38)-(39). We will see that our sufficient condition for absolute stability can be weaker than the one in [13]. For example, if we choose $\alpha_{i j}=\alpha>0, \sigma_{i j}=\sigma>0$, their sufficient condition for absolute stability (see [13, p.231]) can be reduced to $\alpha<1 / 8$ (independent of $\sigma$ ). So if we consider $\alpha=1 / 4$ and $\sigma \geq 1 / \sqrt{8 \pi}$ which satisfy (40) but do not satisfy their sufficient condition, the solution is still absolutely stable. Thus, with the same data as in Example 5.1, but replacing (41) by

$$
\alpha_{i j}=\alpha=1 / 4, \sigma_{i j}=\sigma=1 / \sqrt{8 \pi}, i, j=1,2,
$$

Figure 3 illustrates that the two solutions still approach each other as $t \rightarrow \infty$.

Example 5.4. We demonstrate the synchronization among different layers by choosing parameters satisfying Theorem 4.3. Consider a circulant matrix $\mathbf{W}$, i.e.,

$$
\alpha_{11}=\alpha_{22}, \alpha_{12}=\alpha_{21}, \sigma_{11}=\sigma_{22}, \sigma_{12}=\sigma_{21} .
$$

We choose the same data as in Example 5.1, but replace (41) by

$$
\left(\alpha_{11}, \alpha_{12}, \alpha_{21}, \alpha_{22}\right)=(1,3,3,1),\left(\sigma_{11}, \sigma_{12}, \sigma_{21}, \sigma_{22}\right)=(1,4,4,1),
$$

and the external current by

$$
I_{1}(x, t)=e^{-t}+\sin t+\sin (6 \pi x), I_{2}(x, t)=e^{-2 t}+\sin t+\sin (6 \pi x) .
$$

Then condition (40) and the assumptions in Corollary 4.1 are met, and the synchronization among two layers occurs, i.e.,

$$
\sup _{x \in[0,1]}\left|V_{1}(x, t)-V_{2}(x, t)\right| \rightarrow 0, \text { as } t \rightarrow \infty,
$$

as indicated in Figure 4. However, if we change $\alpha_{i j}$ to, for example,

$$
\left(\alpha_{11}, \alpha_{12}, \alpha_{21}, \alpha_{22}\right)=(40,-30,-30,40) \text {, }
$$

so that (40) does not hold, then the synchrony is lost, as shown in Figure 5. 

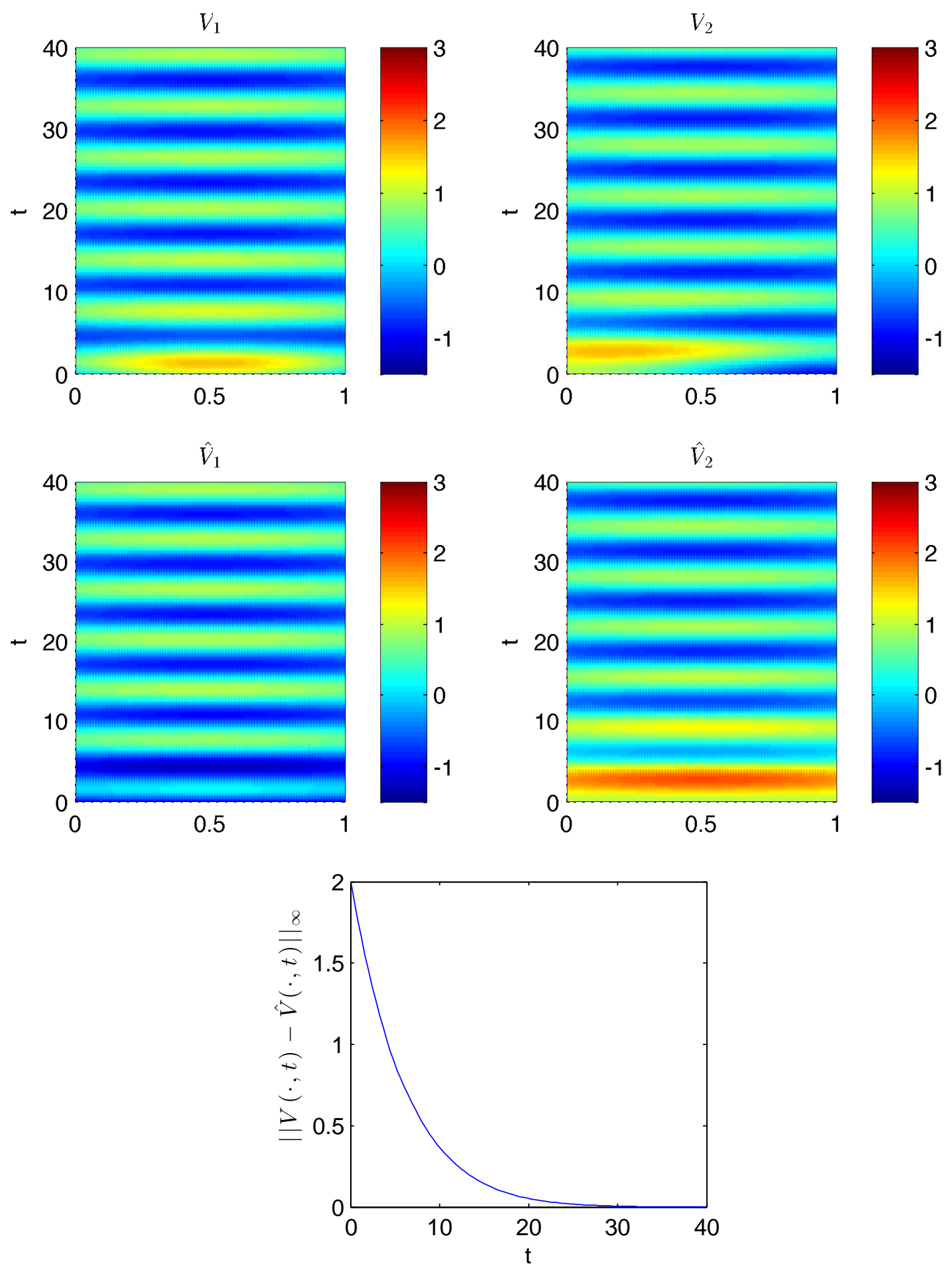

Figure 1: Absolute stability in system (38)-(39). Top row: the solution $\left(V_{1}, V_{2}\right)$ with initial condition $\left(\phi_{1}(x, t), \phi_{2}(x, t)\right)=(\sin \pi x, \cos \pi x), x \in$ $[0,1], t \in[-1 / 10,0]$. Middle row: the solution $\left(\hat{V}_{1}, \hat{V}_{2}\right)$ with initial condition $\left(\hat{\phi}_{1}(x, t), \hat{\phi}_{2}(x, t)\right)=\left(e^{-t}-2, e^{t}\right)$ for $x \in[0,1], t \in[-1 / 10,0]$. Bottom row: the evolution of $\|V(\cdot, t)-\hat{V}(\cdot, t)\|_{\infty}$ with respect to time. 

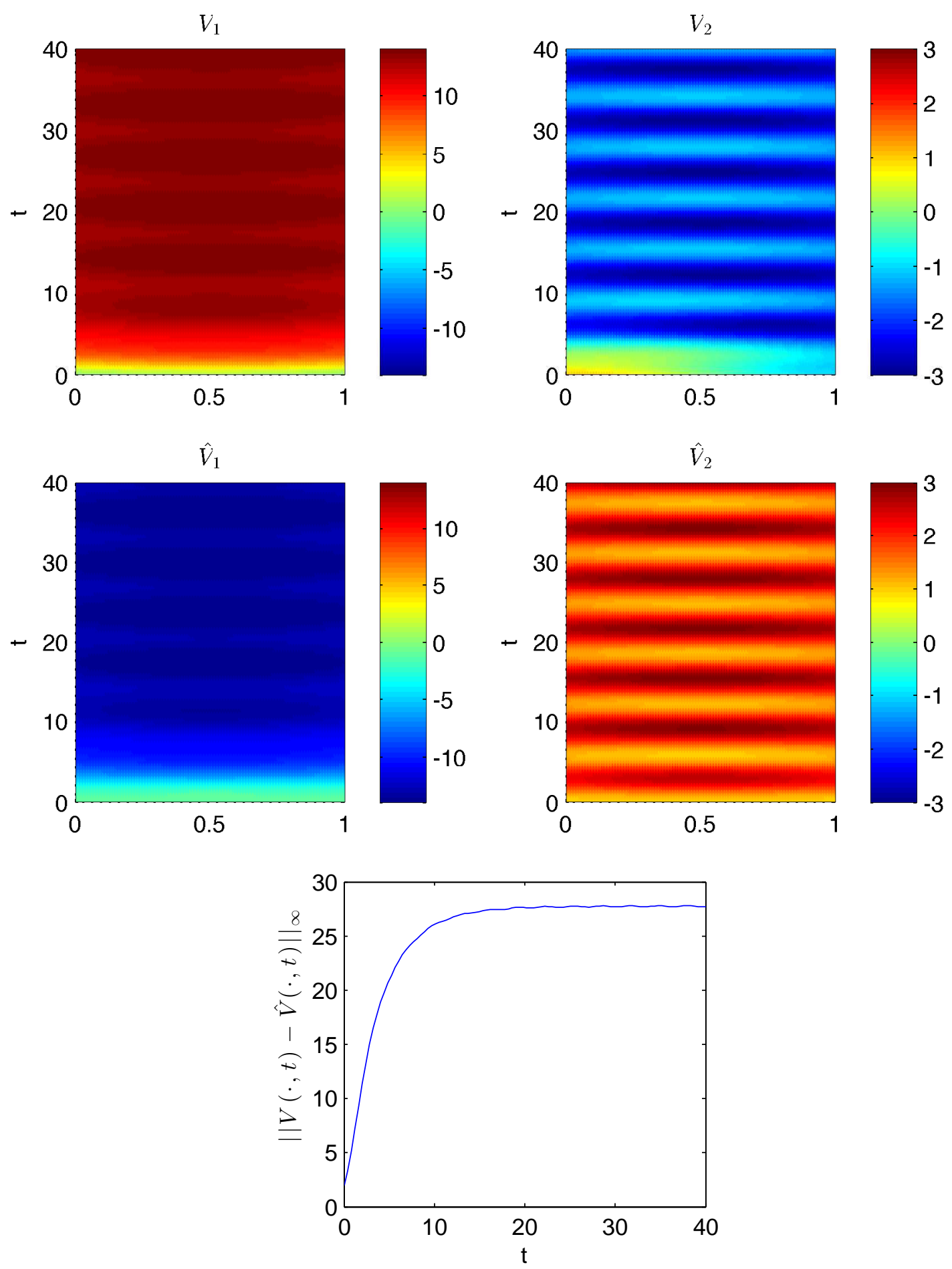

Figure 2: Loss of absolute stability in system (38)-(39). Top row: the solution $\left(V_{1}, V_{2}\right)$ with initial $\operatorname{condition}\left(\phi_{1}(x, t), \phi_{2}(x, t)\right)=(\sin \pi x, \cos \pi x)$, for $x \in[0,1], t \in[-1 / 10,0]$. Middle row: the solution $\left(\hat{V}_{1}, \hat{V}_{2}\right)$ with initial condition $\left(\hat{\phi}_{1}(x, t), \hat{\phi}_{2}(x, t)\right)=\left(e^{-t}-2, e^{t}\right)$, for $x \in[0,1], t \in[-1 / 10,0]$. Bottom row: the evolution of $\|V(\cdot, t)-\hat{V}(\cdot, t)\|_{\infty}$ with respect to time. 

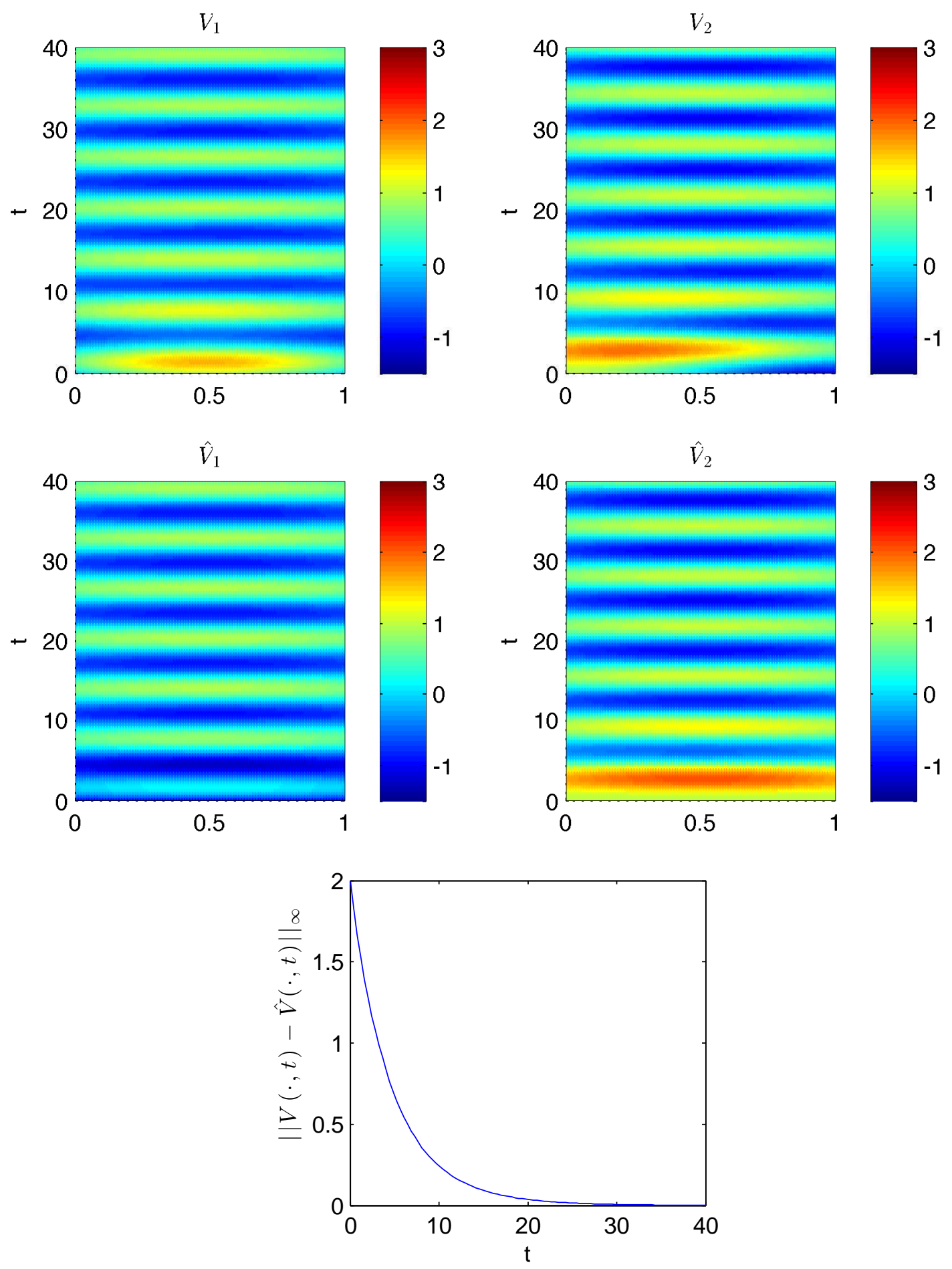

Figure 3: Absolute stability without time delays. Top row: the solution $\left(V_{1}, V_{2}\right)$ with initial condition $\left(\phi_{1}(x, t), \phi_{2}(x, t)\right)=(\sin \pi x, \cos \pi x), x \in$ $[0,1], t \in[-1 / 10,0]$. Middle row: the solution $\left(\hat{V}_{1}, \hat{V}_{2}\right)$ with initial condition $\left(\hat{\phi}_{1}(x, t), \hat{\phi}_{2}(x, t)\right)=\left(e^{-t}-2, e^{t}\right)$, for $x \in[0,1], t \in[-1 / 10,0]$. Bottom row: the evolution of $\|V(\cdot, t)-\hat{V}(\cdot, t)\|_{\infty}$ with respect to time. 

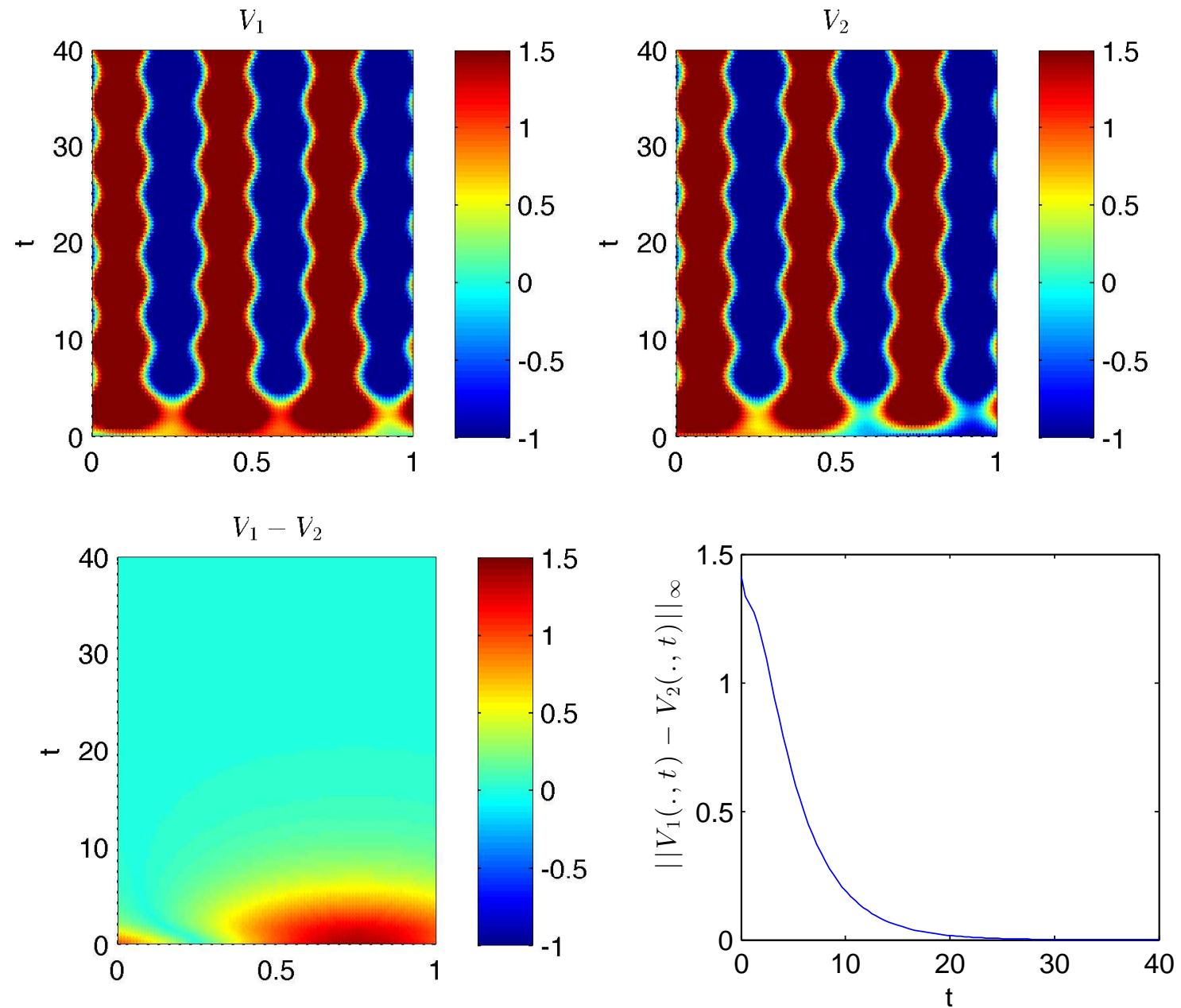

Figure 4: Synchronization in system (38)-(39). Top row: the solution $\left(V_{1}, V_{2}\right)$ with initial condition $\left(\phi_{1}(x, t), \phi_{2}(x, t)\right)=(\sin \pi x, \cos \pi x), x \in$ $[0,1], t \in[-1 / 10,0]$. Bottom row: the difference between $V_{1}$ and $V_{2}$ and evolution of $\left\|V_{1}(\cdot, t)-V_{2}(\cdot, t)\right\|_{\infty}$ with respect to time. 

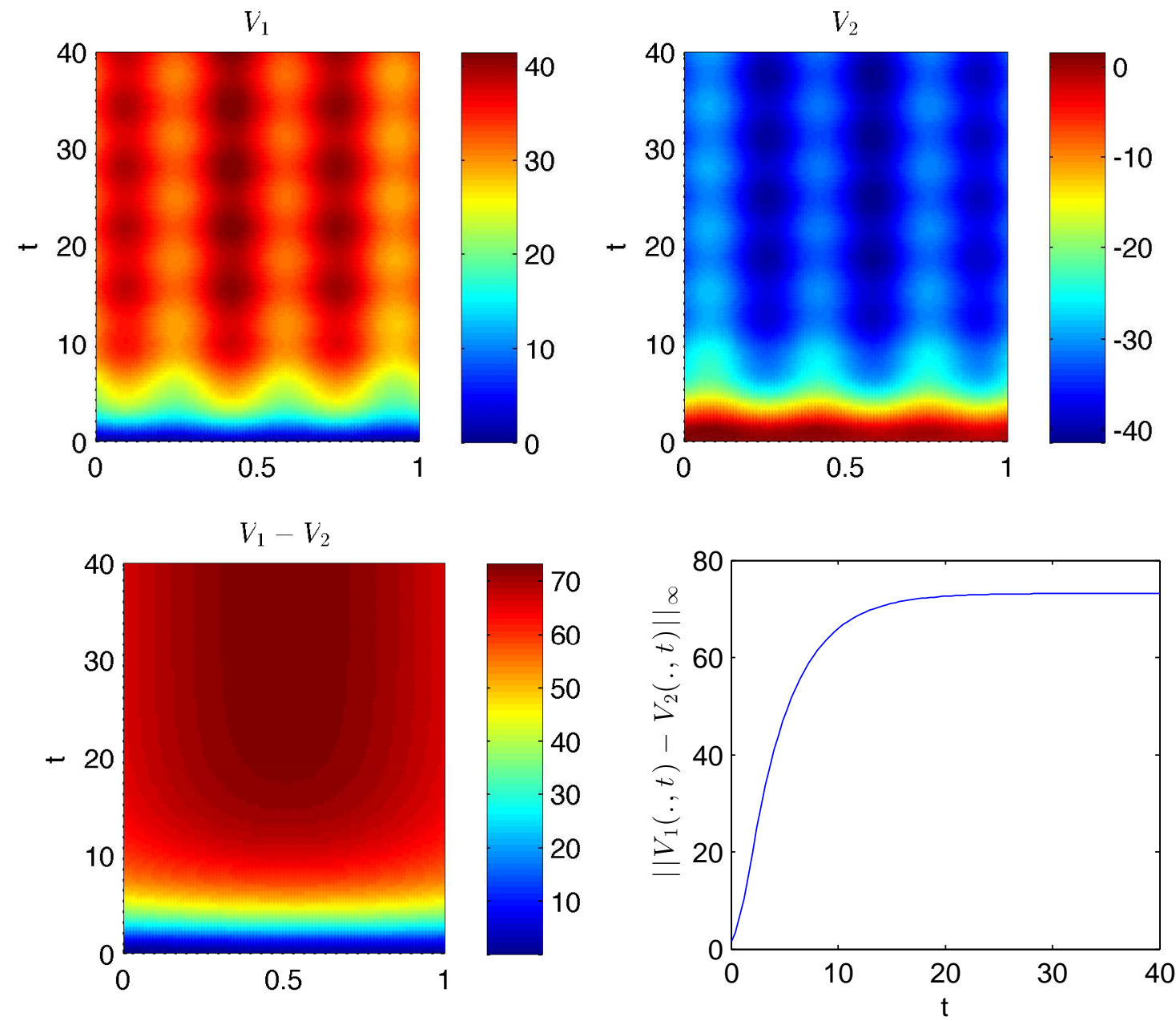

Figure 5: Loss of synchronization in system (38)-(39). Top row: the solution $\left(V_{1}, V_{2}\right)$ with the initial condition $\left(\phi_{1}(x, t), \phi_{2}(x, t)\right)=(\sin \pi x, \cos \pi x)$, for $x \in[0,1], t \in[-1 / 10,0]$. Bottom row: the difference between $V_{1}$ and $V_{2}$ and evolution of $\left\|V_{1}(\cdot, t)-V_{2}(\cdot, t)\right\|_{\infty}$ with respect to time. 


\section{Conclusion}

In this paper, based on the functional differential equation theory, we proved the global existence and uniqueness of classical solutions for a class of neural field models. Through an iteration argument, we derived a sufficient condition for absolute stability of the general solution in the considered systems. Such an assertion was termed all-delay stability or the delay-independent stability, and the related issue has been called for research in [36]. The present analysis allows the underlying spatial domain $\Omega$ to be bounded or unbounded. Our criterion for absolute stability applies to the systems with transmission time delays which are space-dependent. The criterion for absolute stability in previous work [13] applies to systems without delay and depends on an operator norm involving the connectivity matrix function. Our criterion also leads to the globally asymptotical stability of stationary solution for system (1) with space-independent external currents. In addition, synchronization among different layers and within the same layer of the system were established under some assumptions. The analysis can be extended to obtain parallel results in activity-based model with time delays $[11,13]$.

While taking space-dependent delays into account in the neural field models is indeed crucial and practical, it also raises mathematical technicality in understanding the dynamics of the models. The present approach and results are expected to contribute toward further understanding on these important models.

Acknowledgements. The authors are grateful to the reviewers for their comments which have led to improve the presentation. C.-Y. Kao is partially supported by NSF DMS-1318364. C.-W. Shih and C.-H. Wu are partially supported by the Ministry of Science and Technology, R.O.C.

[1] S.-I. Amari, Homogeneous nets of neuron-like elements, Biol. Cybernet., 17 (1975), pp. 211-220.

[2] S.-I. Amari, Dynamics of pattern formation in lateral-inhibition type neural fields, Biol. Cybernet., 27 (2) (1977), pp. $77-87$.

[3] F. M. Atay and A. Hutt, Stability and bifurcations in neural fields with finite propagation speed and general connectivity, SIAM J. Appl. Math., 65 (2) (2005), pp. 644-666.

[4] F. M. Atay and A. Hutt, Neural fields with distributed transmission speeds and long-range feedback delays, SIAM J. Appl. Dyn. Syst., 5 (4) (2006), pp. 670-698.

[5] P. C. Bressloff, Traveling fronts and wave propagation failure in an inhomogeneous neural network, Physica D, 155 (2001), pp. $83-100$.

[6] P. C. Bressloff, Spatiotemporal dynamics of continuum neural Fields, J. Phys. A, 45 (2012), 033001.

[7] S. Coombes, Waves, bumps, and patterns in neural fields theories, Biol. Cybernet., 93 (2) (2005), pp. 91-108.

[8] P. J. Davis, Circulant Matrices, Wiley-Interscience, New York, 1979.

[9] G. B. Ermentrout, Neural networks as spatio-temporal pattern-forming systems, Reports on Progress in Physics, 61 (1998), pp. $353-430$.

[10] G. B. Ermentrout and J. B. McLeod, Existence and uniqueness of travelling waves for a neural network, Proceedings of the Royal Society of Edinburgh, 123A (1993), pp. 461-478.

[11] O. Faugeras, R. Veltz, and F. Grimbert, Persistent neural states: Stationary localized activity patterns in nonlinear continuous n-population, q-dimensional neural networks, Neural Comput., 21 (2009), pp. 147-187.

[12] G. Faye and O. Faugeras, Some theoretical and numerical results for delayed neural field equations, Physica D, 239 (2010), pp. 561-578.

[13] O. Faugeras, F. Grimbert, and J.-J. Slotine, Absolute stability and complete synchronization in a class of neural fields models, SIAM J. Appl. Math., 69 (2008), pp. 205-250.

[14] Y. Guo and C. C. Chow, Existence and stability of standing pulses in neural networks: I. Existence, SIAM J. Appl. Math., 4 (2005), pp. 217248.

[15] Y. Guo and C. C. Chow, Existence and stability of standing pulses in neural networks: II. Stability, SIAM J. Appl. Dyn. Syst., 4 (2005), pp. 249-281.

[16] S. Haeusler and W. Maass, A statistical analysis of information-processing properties of lamina-specific cortical microcircuits models, Cerebral Cortex, 17 (2007), pp. 149162.

[17] J. Hale, Diffusive coupling, dissipation, and synchronization, J. Dynamics and Differential Equations, Vol. 9, No. 1 (1997), 1-52.

[18] J. K. Hale and S. M. V. Lunel, Introduction to Functional Differential Equations, Springer Verlag, 1993.

[19] F. Hirsch and G. Lacombe. Elements of Functional Analysis. New York: Springer, 1999.

[20] B. H. Jansen, G. Zouridakis, and M. E. Brandt, A neurophysiologically-based mathematical model of flash visual evoked potentials, Biol. Cybernet., 68 (1993), pp. 275-283.

[21] V. K. Jirsa, Connectivity and dynamics of neural information processing, Neuroinformatics, 2 (2004), pp. 183-204.

[22] Z. P. Kilpatrick, S. E. Folias, and P. C. Bressloff,Traveling pulses and wave propagation failure in inhomogeneous neural media, SIAM J. Appl. Dyn. Syst., 7 (2008), pp. 161-85.

[23] K. Kishimoto and S. Amari, Existence and stability of local excitations in homogeneous neural fields, J. Math. Biology, 7 (1979), pp. 303-318.

[24] C. L. Laing, W. C. Troy, B. Gutkin, and G. B. Ermentrout, Multiple bumps in a neuronal model of working memory, SIAM J. Appl. Math., 63 (2002), pp. 62-97.

[25] C. R. Laing and W. C. Troy,Two-bump solutions of Amari-type models of neuronal pattern formation, Physica D, 178 (2003), pp. $190-218$.

[26] G. S. Medvedev, Electrical coupling promotes fidelity of responses in the networks of model neurons, Neural Comput., 2 (2009), pp. 30573078.

[27] D. J. Pinto and G. B. Ermentrout, Spatially structured activity in synaptically coupled neuronal networks: I. Travelling fronts and pulses, SIAM J. Appl. Math., 62 (2001), pp. 206-225. 
[28] R. Potthast and P. B. Graben, Existence and properties of solutions for neural field equations, Math. Meth. Appl. Sci., 33 (2010), pp. 935-949.

[29] C.-W. Shih and J.-P. Tseng, Global synchronization and asymptotic phases for a ring of identical cells with delayed coupling, SIAM J. Math. Anal., 43 (2011), pp. 1667-1697.

[30] C.-W. Shih and J.-P. Tseng, A general approach to synchronization of coupled cells, SIAM J. Appl. Dyn. Syst., 12 (2013), pp. $1354-1393$.

[31] A. van Rotterdam, F. H. Lopes da Silva, J. van den Ende, M. A. Viergever, and A. J. Hermans, A model of the spatial-temporal characteristics of the alpha rhythm, Bulletin of Mathematical Biology, 44(2) (1982), pp. 283-305.

[32] S. A. Van Gils, S. G. Janssens, Y. A. Kuznetsov, and S. Visser, On local bifurcations in neural field models with transmission delays, J. Math. Biol., 66 (2013) pp. 837-87.

[33] N. A. Venkov, S. Coombes, and P. C. Matthews, Dynamic instabilities in scalar neural field equations with space-dependent delays, Physica D, 232 (2007), pp. 1-15.

[34] A. M. Thomson and A. P. Bannister, Interlaminar connections in the neocortex, Cerebral Cortex, 13 (2003), pp. 514.

[35] R. Veltz and O. Faugeras, Local/global analysis of the stationary solutions of some neural field equations, SIAM J. Appl. Dyn. Syst., 9 (2010), pp. 954-998.

[36] R. Veltz and O. Faugeras, Stability of the stationary solutions of neural field equations with propagation delays, J. Mathematical Neuroscience, $1: 1$ (2011), pp. 1-28.

[37] H. R. Wilson and J. D. Cowan, Excitatory and inhibitory interactions in localized populations of model neurons, Biophys. J., 12 (1972), pp. $1-24$.

[38] H. R. Wilson and J. D. Cowan, A mathematical theory of the functional dynamics of cortical and thalamic nervous tissue, Biol. Cybernet., 13 (2) (1973), pp. 55-80. 
Fig. 1a

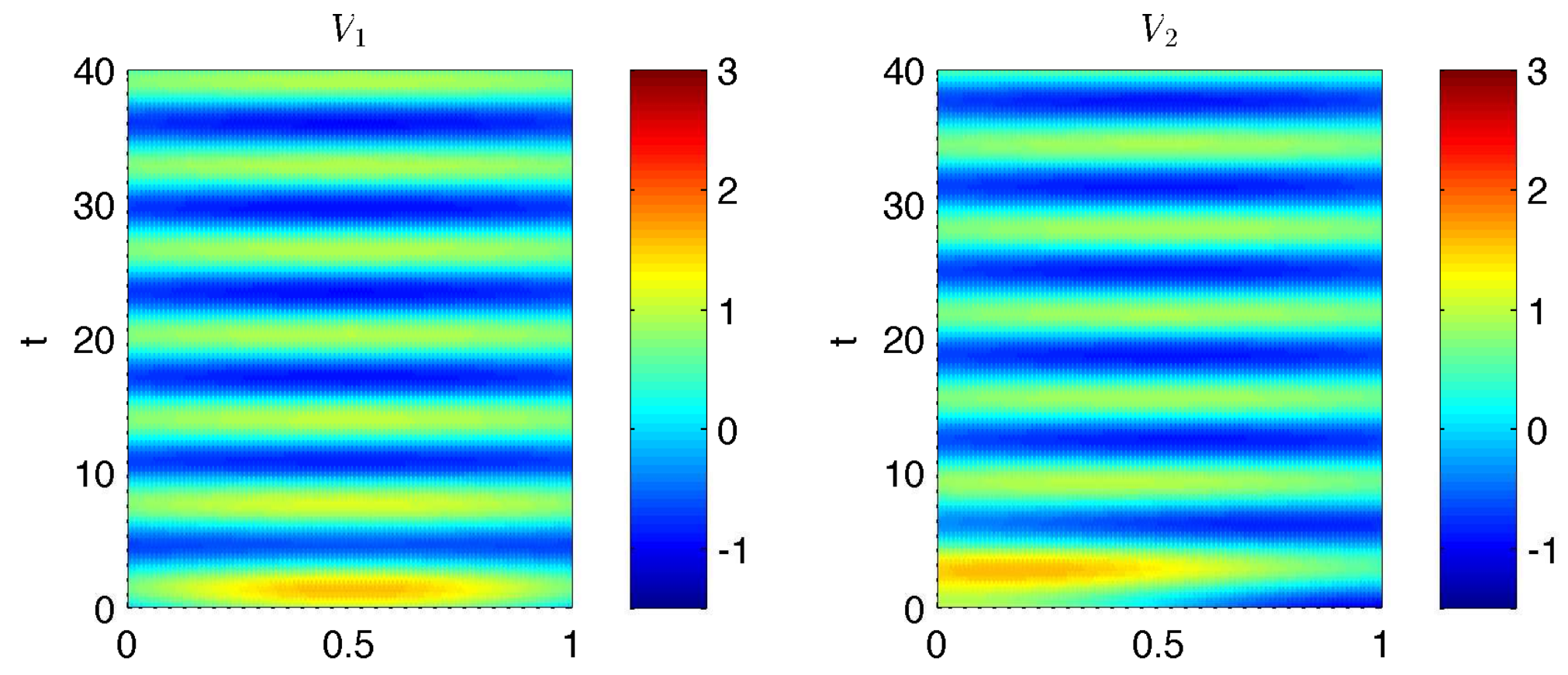


Fig. 1b
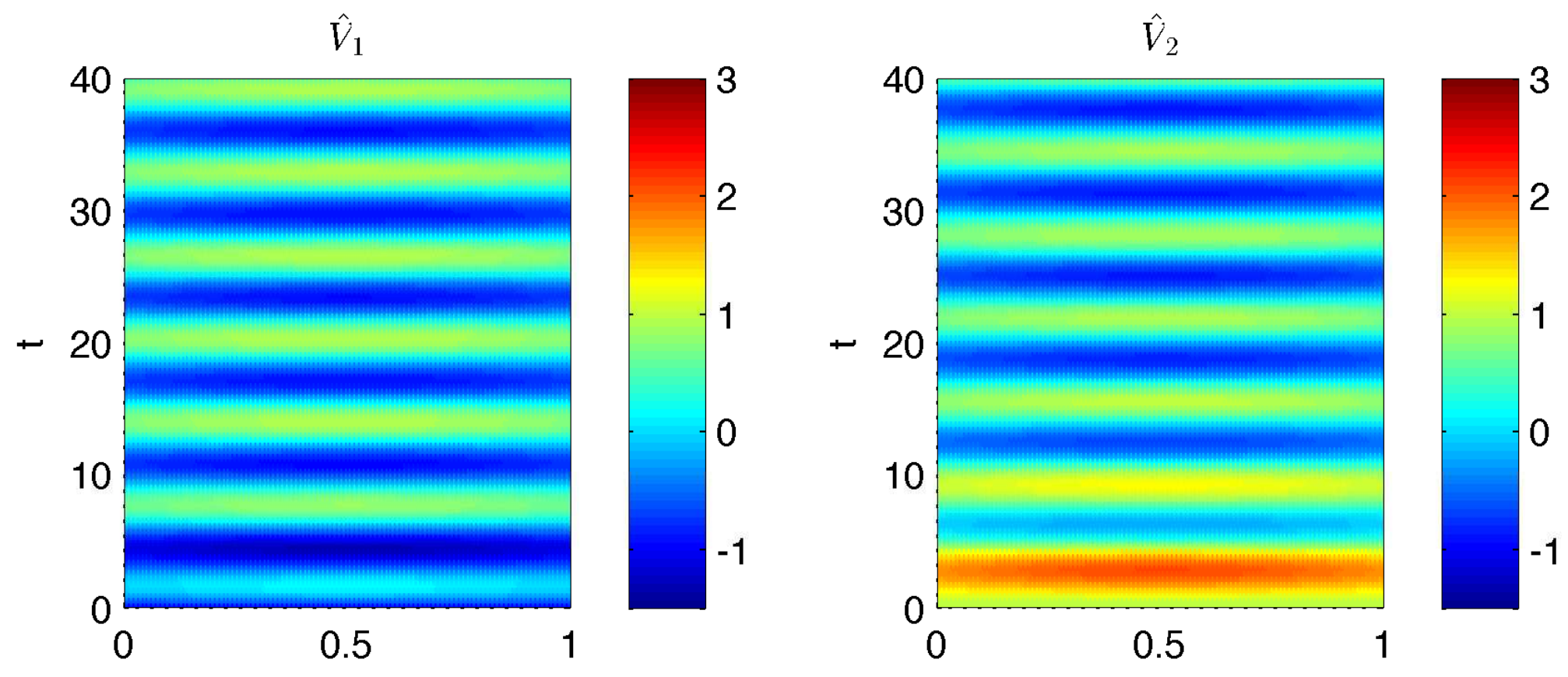
Fig. 1c

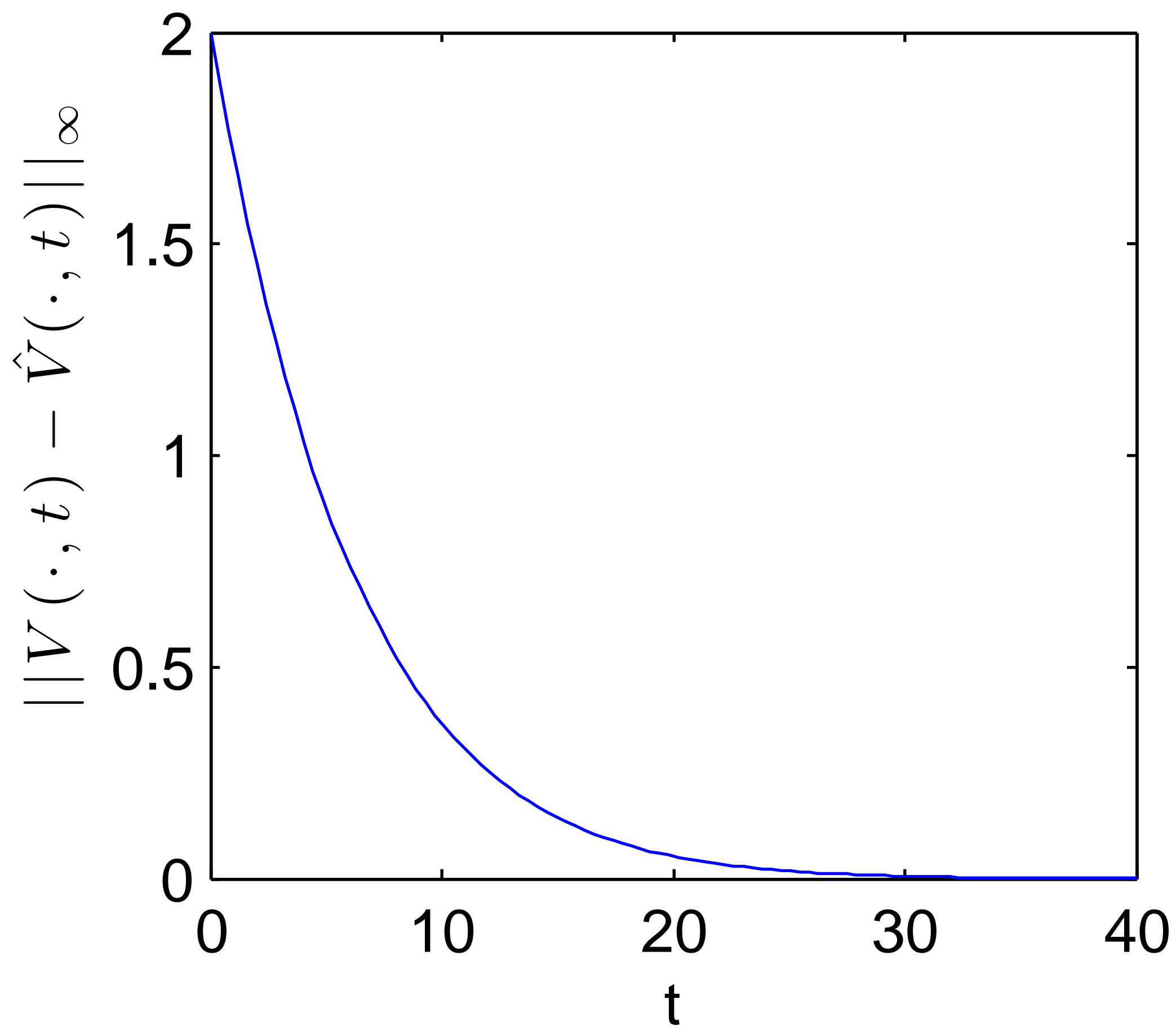


Fig. 2 a
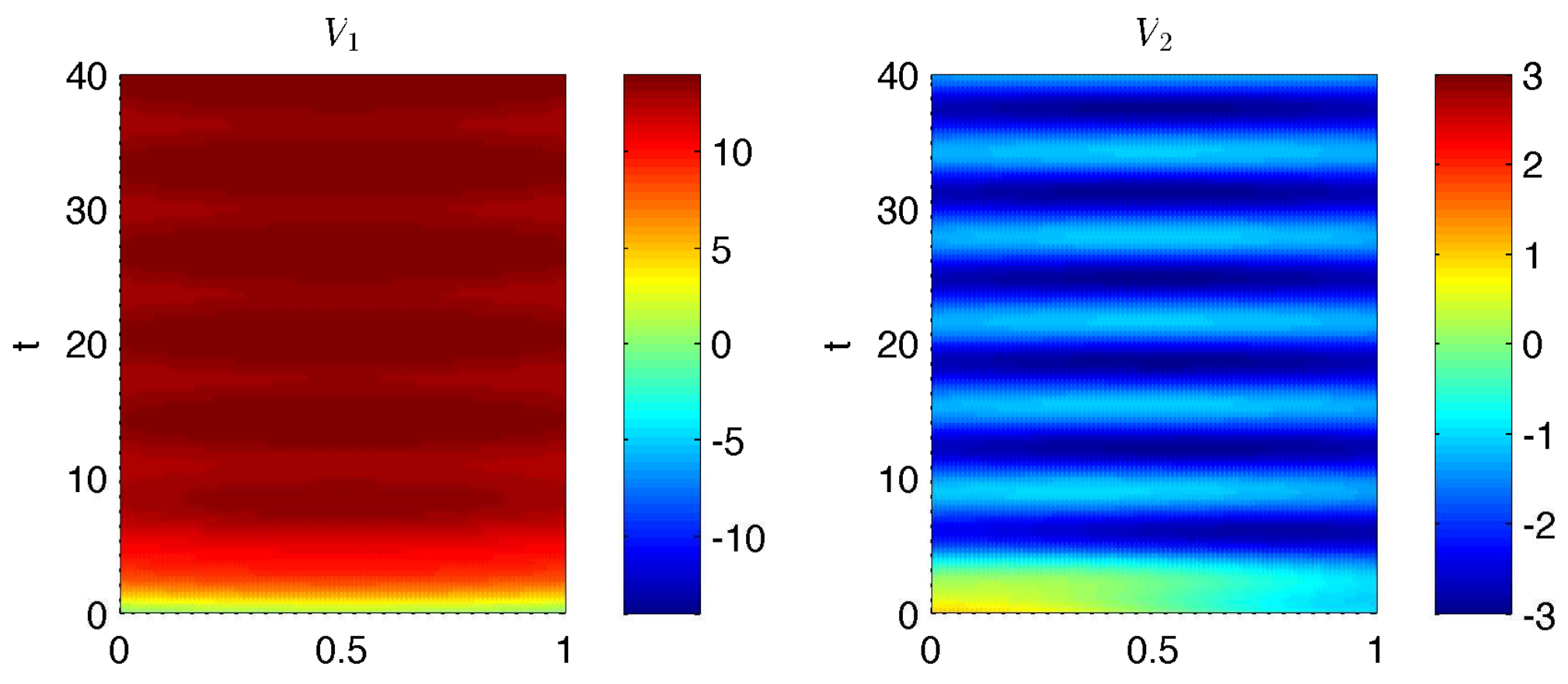
Fig. $2 b$
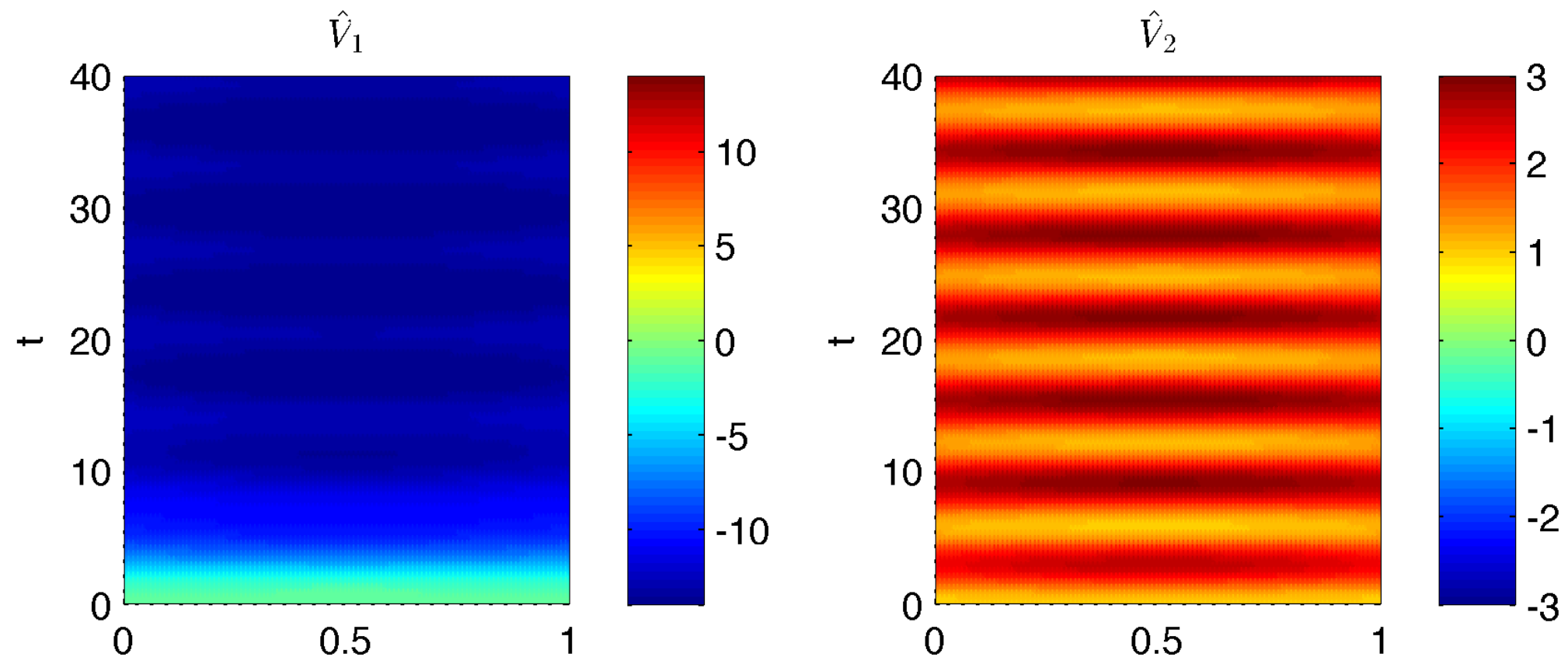
Fig. 2c

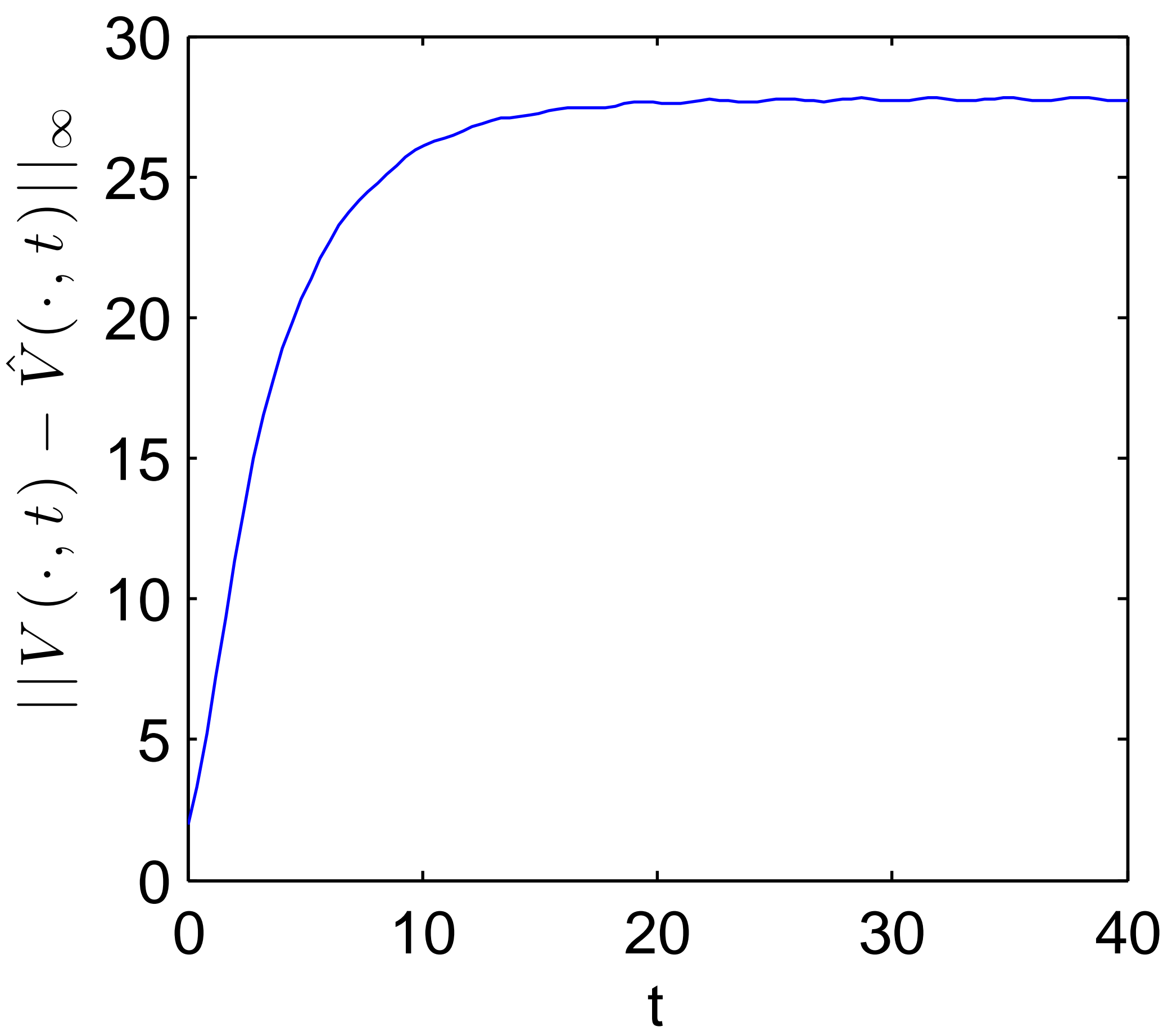


Fig. 3a
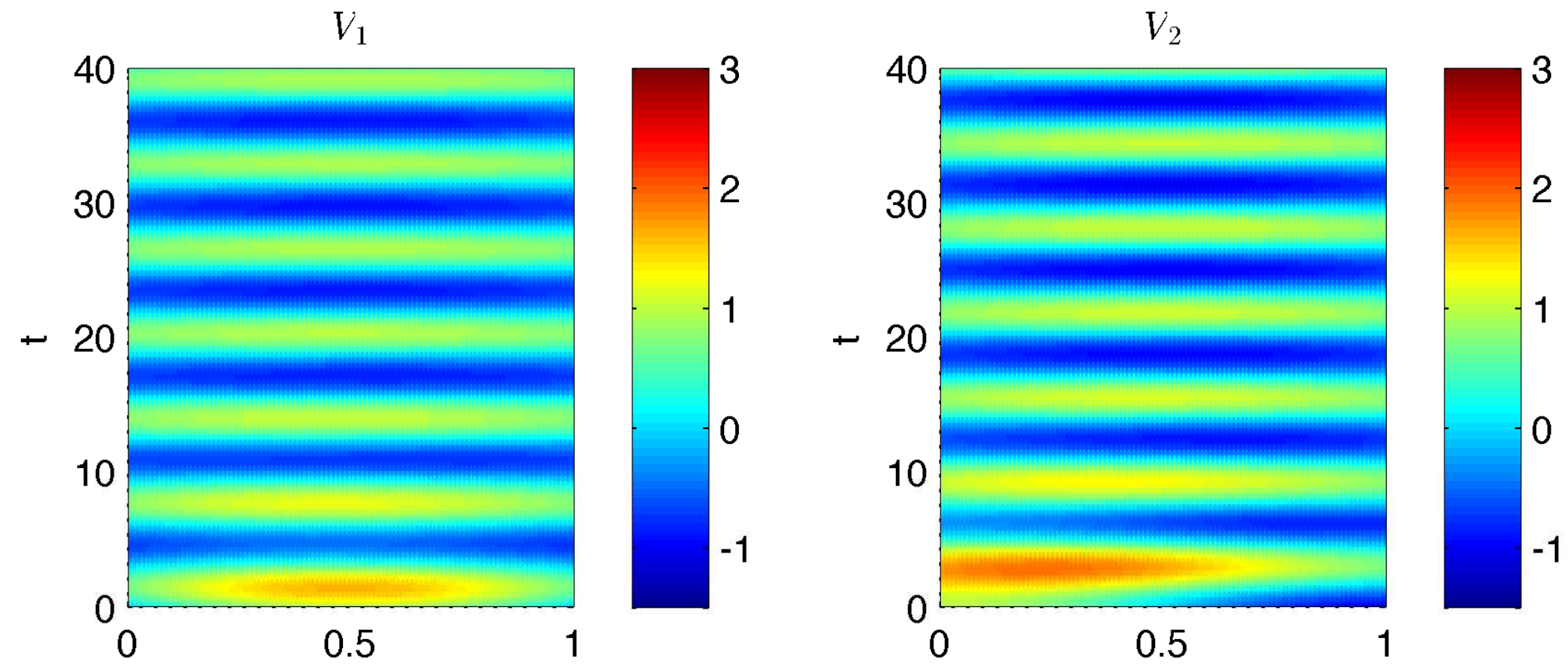
Fig. 3b

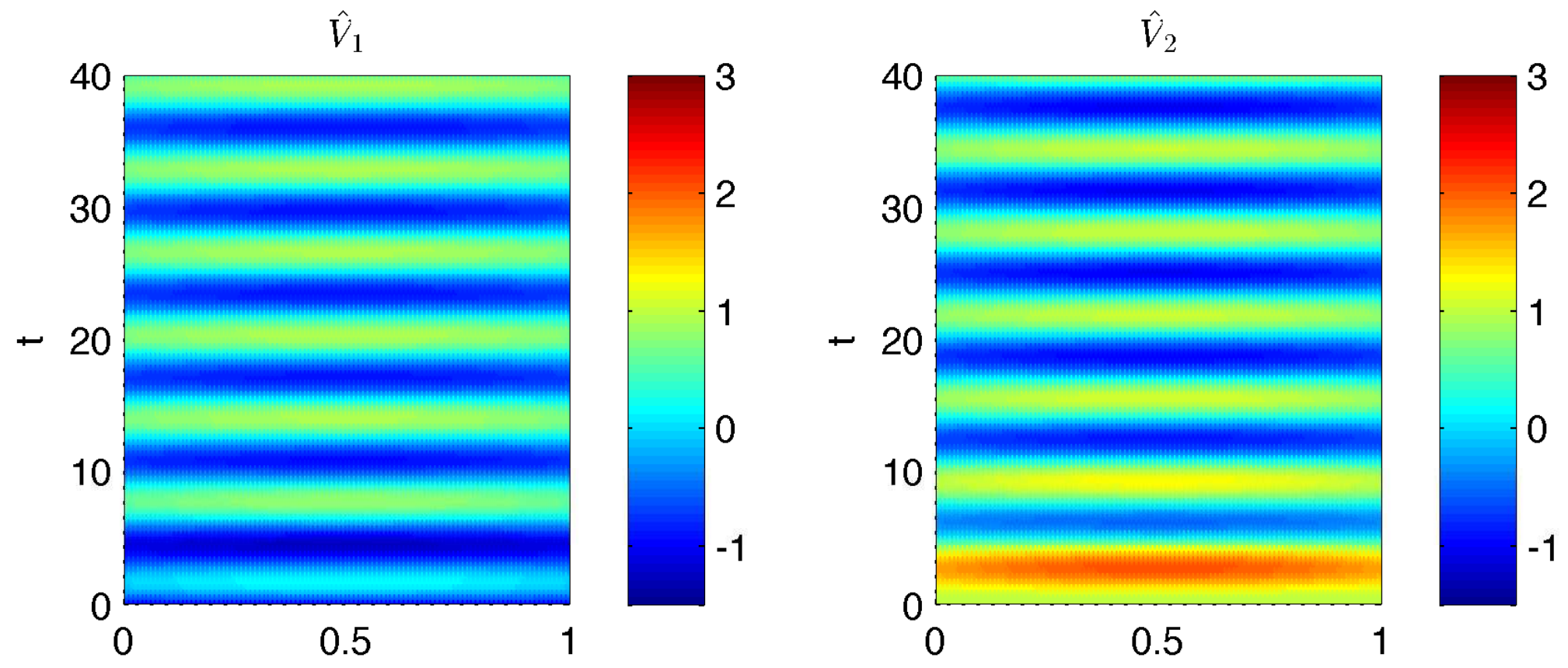


Fig. $4 a$
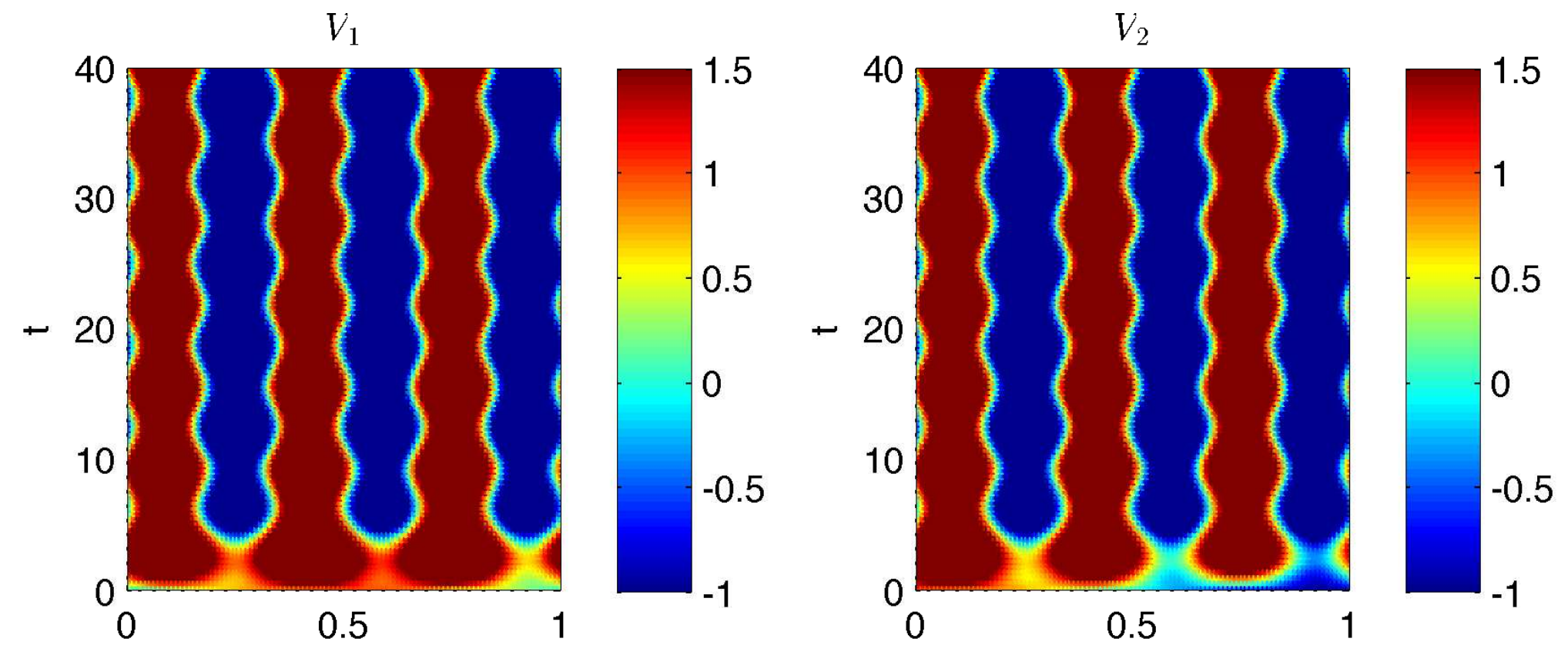
Fig. $4 b$

$V_{1}-V_{2}$
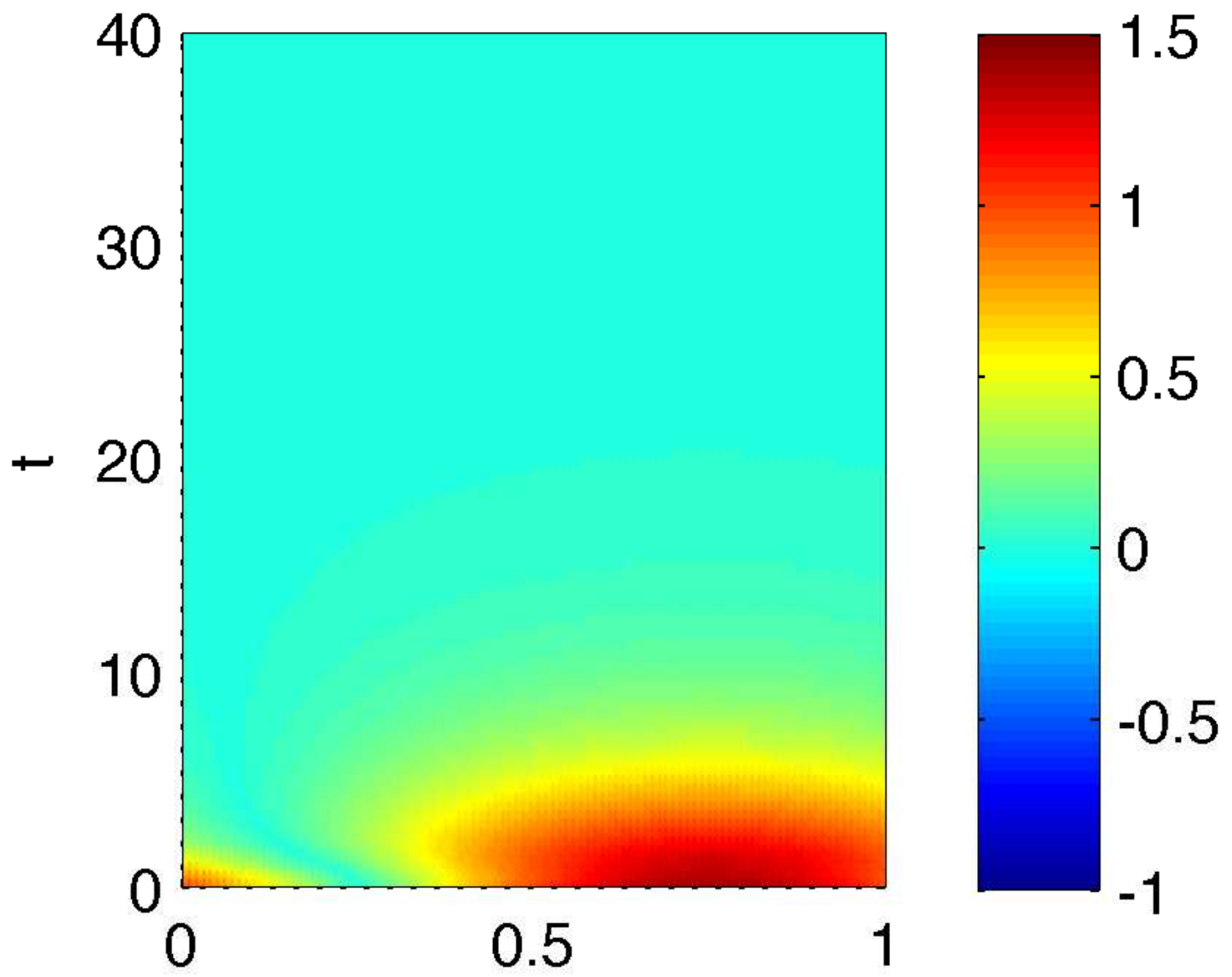
Fig. 4bb

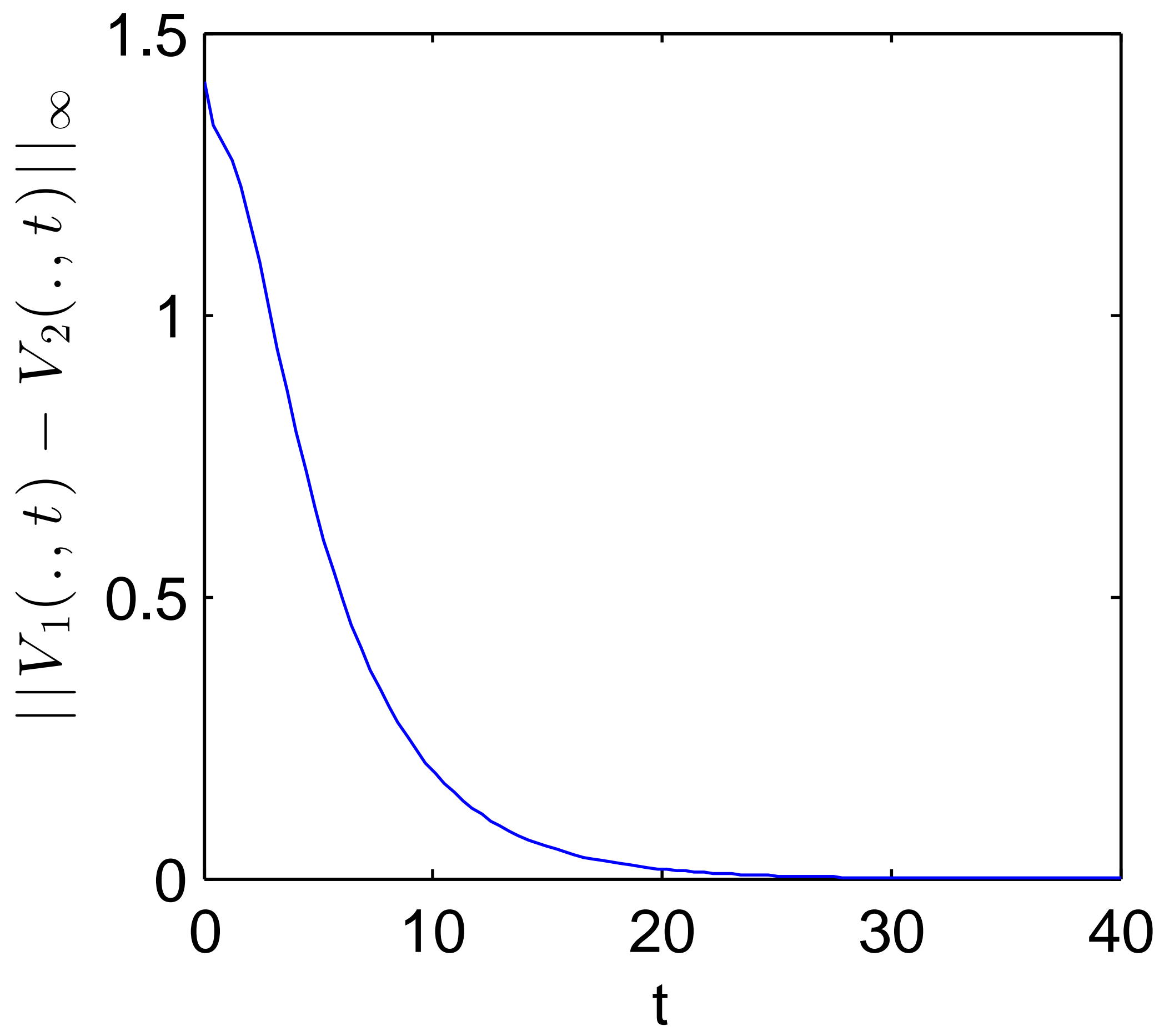


Fig. 5a

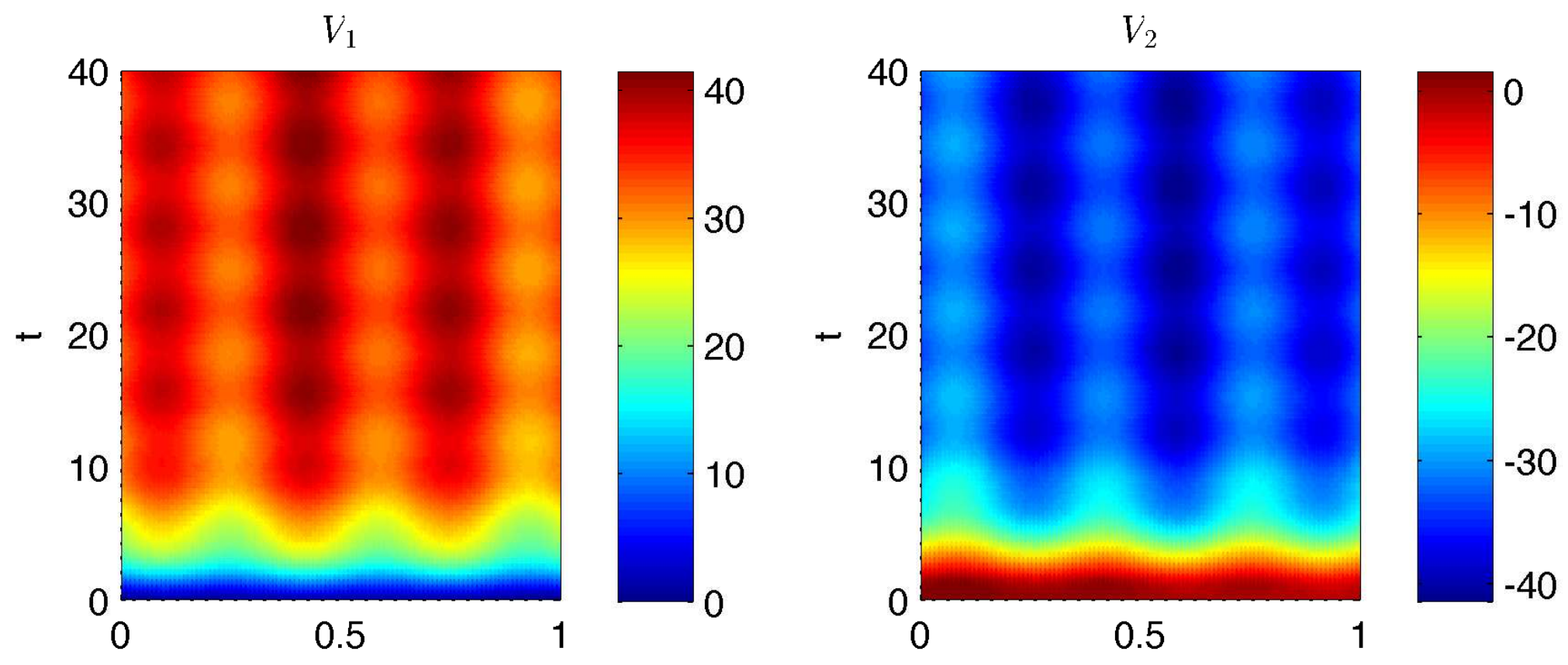


Fig. 5b

$$
V_{1}-V_{2}
$$

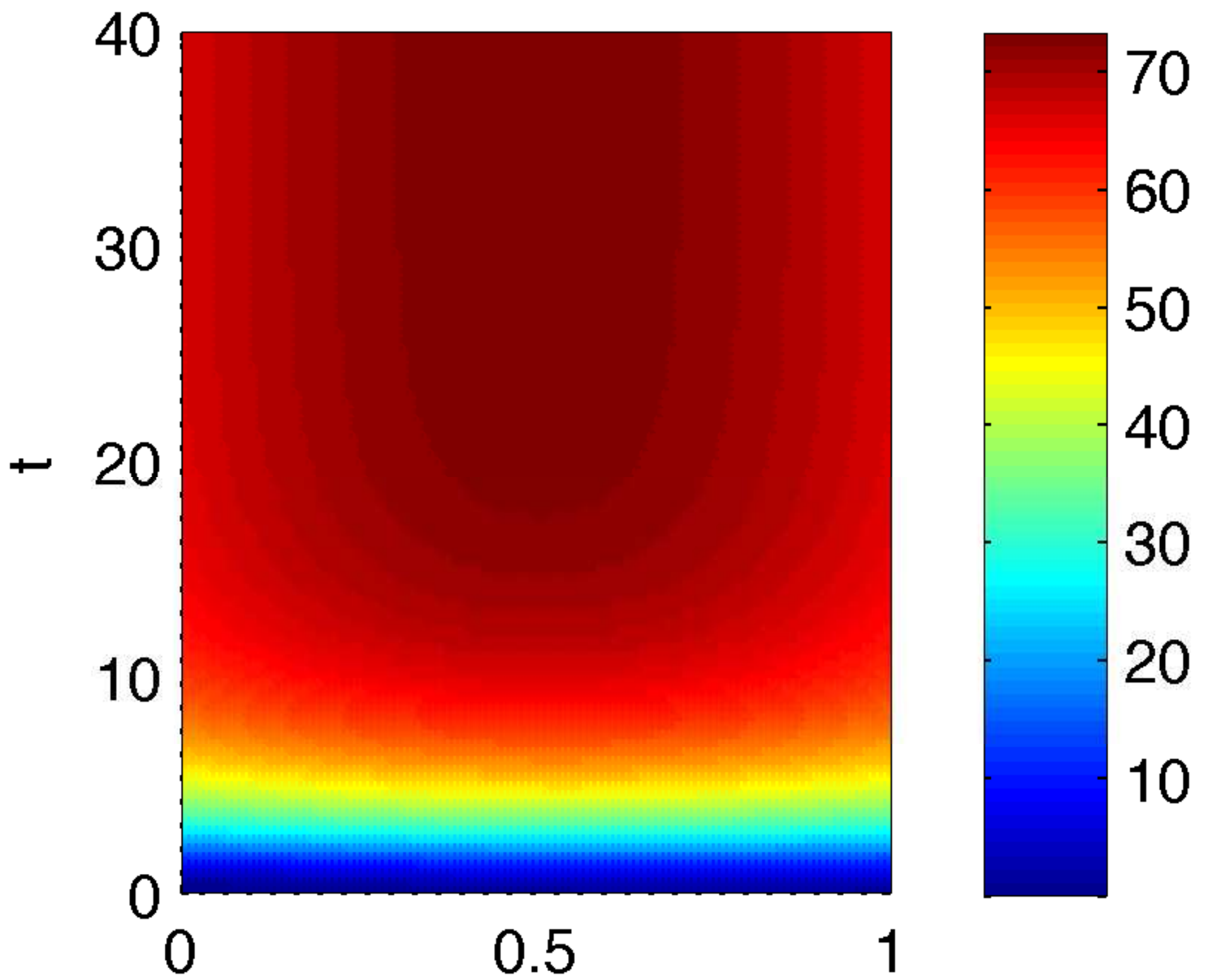


Fig. 5bb

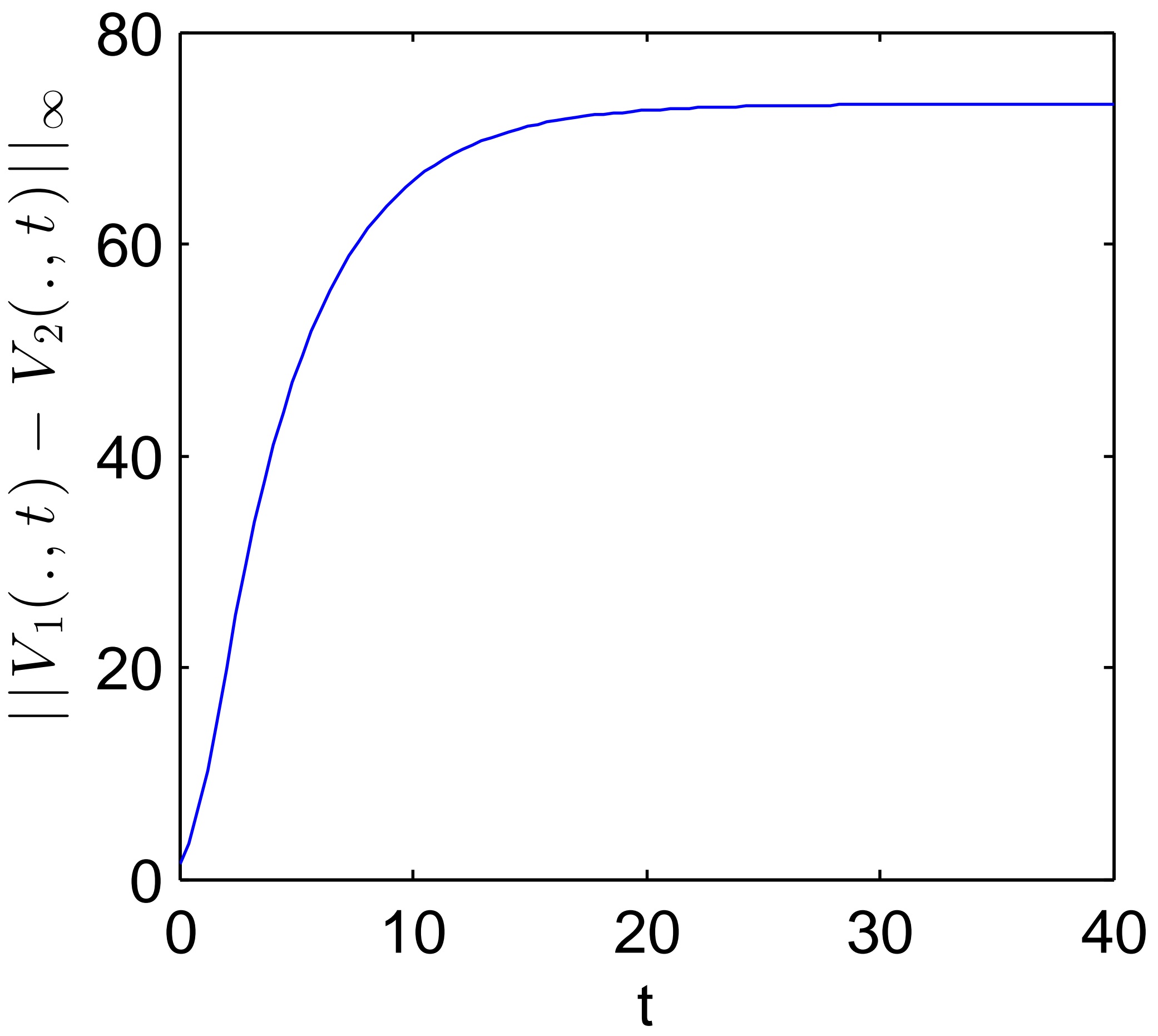

